\title{
Minimization of Fuel Costs and Gaseous Emissions of Electric Power Generation by Model Predictive Control
}

\author{
A. M. Elaiw, ${ }^{1,2}$ X. Xia, ${ }^{3}$ and A. M. Shehata ${ }^{2}$ \\ ${ }^{1}$ Department of Mathematics, Faculty of Science, King Abdulaziz University, P.O. Box 80203, Jeddah 21589, Saudi Arabia \\ ${ }^{2}$ Department of Mathematics, Faculty of Science, Al-Azhar University, Assiut 71511, Egypt \\ ${ }^{3}$ Centre of New Energy Systems, Department of Electrical, Electronic and Computer Engineering, University of Pretoria, \\ Pretoria 0002, South Africa
}

Correspondence should be addressed to A. M. Elaiw; a_m_elaiw@yahoo.com

Received 15 January 2013; Revised 12 March 2013; Accepted 26 March 2013

Academic Editor: Alex Elias-Zuniga

Copyright ( $) 2013$ A. M. Elaiw et al. This is an open access article distributed under the Creative Commons Attribution License, which permits unrestricted use, distribution, and reproduction in any medium, provided the original work is properly cited.

\begin{abstract}
The purpose of this paper is to present a model predictive control (MPC) approach for the periodic implementation of the optimal solutions of two optimal dynamic dispatch problems with emission and transmission line losses. The first problem is the dynamic economic emission dispatch (DEED) which is a multiobjective optimization problem which minimizes both fuel cost and pollutants emission simultaneously under a set of constraints. The second one is the profit-based dynamic economic emission dispatch (PBDEED) which is also a multi-objective optimization problem which maximizes the profit and minimizes the emission simultaneously under a set of constraints. Both the demand and energy price are assumed to be periodic and the total transmission loss is assumed to be a quadratic function of the generator power outputs. We assume that there are certain disturbances or uncertainties in the execution of the optimal controller and in the forecasted demand. The convergence and robustness of the MPC algorithm are demonstrated through the application of MPC to the DEED and PBDEED problems with five-unit and six-unit test systems, respectively.
\end{abstract}

\section{Introduction}

Reducing fuel cost in electric-power-generating plants has received considerable attention in recent years. Dynamic economic dispatch (DED) is a real-time power system problem which is used to schedule the committed electric-powergenerating units' outputs so as to meet the load demand over a dispatch period at minimum operating cost while satisfying ramp rate constraints and other constraints (see, e.g., [1-11]). Since the ramp rate constraints couple the time intervals, the DED problem is a difficult optimization problem. Since the DED problem was introduced, several optimization techniques and procedures have been used for solving the DED problem with complex objective functions or constraints (see the review paper [1]). After deregulation of the electric power system, the objective of the dynamic dispatch problem has been changed from cost minimization to profit maximization and the problem is known as profitbased dynamic economic dispatch (PBDED) problem [11].

The emission of gaseous pollutants including $\mathrm{SO}_{2}, \mathrm{NO}_{x}$, $\mathrm{CO}$, and $\mathrm{CO}_{2}$ from fossil-fuel-fired thermal plants affects the human health directly or indirectly. Therefore, electric utilities and generation companies (GENCOs) are requested to reduce emission from their plants. As a result of public awareness of environmental protection, diverse compliance strategies have emerged. These strategies include installation of pollutant cleaning, switching to low-emission fuels, replacement of the aged fuel burners with cleaner ones, and emission dispatching [12]. The last strategy is usually preferred to the existing systems because it is easy to implement and requires less additional cost.

The emission can be taken into the DED problem by minimizing both fuel cost and emission simultaneously under load demand constraint, ramp rate constraint, and 
other constraints, resulting in a multiobjective optimization problem. This problem is referred to as dynamic economic emission dispatch (DEED) [13-15]. The emission can also be considered into the PBDED problem by formulating the profit-based dynamic economic emission dispatch (PBDEED) problem which is also a multi-objective optimization problem which maximizes the profit and minimizes the emission simultaneously under ramp rate constraint and other constraints.

Recently, many optimization techniques have been used to solve the DEED problem with complex objective functions or constraints (see, e.g., [13-18]). In [16], by assuming that the decision maker has goals for each of the two objective functions, the multi-objective optimization problem is transformed into a single-objective optimization by the goalattainment method and is solved by particle swarm optimization (PSO) method. In [17], it was assumed that the decision maker has a fuzzy goal for each of the objective functions. The optimal noninferior generation schedule is determined by the evaluation-programming- (EP-) based fuzzy satisfying method. In [13], the multi-objective optimization problem is solved by nondominated sorting genetic algorithm-II. In [18], the multi-objective optimization problem is converted into a single-objective optimization and is solved by an improved pattern-search- (PS-) based algorithm. Pandit et al. [14] proposed an improved bacterial foraging algorithm for solving the DEED problem. Group search optimizer with multiple producers has been used to solve the DEED problem in [15].

In [13-18], the main attention focuses on finding the optimal dispatch over a fixed time horizon and the periodic implementation of such an optimal dispatch solution has been though regarded as straightforward and thus left to the practitioners. This periodicity assumption comes from the fact that the demand is periodic due to cyclic consumption behavior and seasonal changes [2]. From a control theoretical point of view, both the DEED and PBDEED formulations provide only open-loop optimal solutions to the generation dispatch problem; that is, the optimal solutions are predetermined before actual execution, and there is no measurement on the system states which is fed back to the optimization model. Therefore, a closed-loop control by the MPC method is introduced in this paper so that the measurement of states can be fed back to the optimization model, and the optimal solution is updated according to the feedback information at each time step. The MPC method has been successfully applied in power systems in [2, 19-22]. MPC has many advantages including its facility of handling constraints, being able to use simple models, and its closedloop stability and inherent robustness. Moreover, MPC solves the optimal control problem online for the current state of the plant which is a mathematical programming problem and is much simpler than determining the feedback solution by dynamic programming [2].

MPC has been proposed for the periodic implementation of the optimal solutions of the DEED and PBDEED problems in [20]. However, the transmission line losses were not included in the DEED and PBDEED formulations. The system loss is a very essential factor to be considered in the power system analysis. Particularly, while the DEED and PBDEED are concerned, the system loss will impose a great impact on optimal pattern of the real power generations at different power plants. In the present paper we propose an MPC approach for the periodic implementation of the optimal solutions of the DEED and PBDEED problems taking into account the transmission losses. The transmission losses will be expressed as a quadratic function of the generator's power outputs.

The remainder of this paper is organized as follows. In Section 2, we introduce the DEED and PBDEED problems under regulated and deregulated power systems. In Section 3, we outline an MPC approach for the DEED problem and summarize the main results obtained in [2]. The simulation results for the application of MPC to the DEED and PBDEED problems are given in Section 4. The last section is the conclusion. Throughout the paper, the following notations and definitions will be used. For a sampling period $T$, the dynamic dispatch problem is considered over dispatch intervals, $[i T,(i+N) T))$, where the optimization is considered, for $i \geq 0$ and NT is the dispatch period. For simplicity, we make the convention throughout the paper that $[i, j)$ denotes the time interval $[i T, j T)$. For any $m \geq 0, k \geq 1$ define $\mathbf{P}^{m}=\left(P_{1}^{1+m}\right.$, $P_{2}^{1+m}, \ldots, P_{n}^{1+m}, P_{1}^{2+m}, P_{2}^{2+m}, \ldots, P_{n}^{2+m}, \ldots, P_{1}^{N+m}, P_{2}^{N+m}, \ldots$, $\left.P_{n}^{N+m}\right)$, and $P^{k}=\left(P_{1}^{k}, P_{2}^{k}, \ldots, P_{n}^{k}\right)^{\prime}$. Define $\mathbf{U}=\left(u_{1}^{1}\right.$, $\left.u_{2}^{1}, \ldots, u_{n}^{1}, u_{1}^{2}, u_{2}^{2}, \ldots, u_{n}^{2}, \ldots, u_{1}^{N-1}, u_{2}^{N-1}, \ldots, u_{n}^{N-1}\right)$ and $D=$ $\left(D^{1}, D^{2}, \ldots, D^{N}\right)^{\prime}$. The total fuel cost and emission from all units and over the dispatch period $[m, m+N)$ are denoted by $C\left(\mathbf{P}^{m}\right)$ and $E\left(\mathbf{P}^{m}\right)$, respectively. The demand $D^{t}$ and the energy price $S P^{t}$ are assumed to be periodic with period $N$.

\section{Optimal Dynamic Dispatch with Emission}

In this section we formulate both DEED and PBDEED problems. Under regulated power systems utilities have the objective to minimize the fuel cost and are obliged to serve all customers and meet all demands. After deregulation and restructuring of the electric power systems, GENCO has the aim to maximize its own profit. In this case, GENCO is not obliged to meet all demands but may sell its energy at less than the system's forecasted demand equilibrium if this will maximize its profit.

2.1. Dynamic Economic Emission Dispatch. It is well known that the fuel cost and the amount of emission conflict with each other. Minimization of fuel cost maximizes the amount of emission and vice versa. Therefore it is necessary to find out an operating point that strikes a balance between fuel cost and emission. This can be done by formulating the DEED problem which is a multi-objective optimization problem with two conflicting objectives, the fuel cost and emission.

The following objectives and constraints are taken into account in the formulation of the DEED problem.

Cost. The fuel cost curves are assumed to be a quadratic function of the generator's active power output. Therefore, 
the total fuel cost over the dispatch period $[0, N]$ is given by [15]

$$
C\left(\mathbf{P}^{0}\right)=\sum_{t=1}^{N} \sum_{i=1}^{n} C_{i}\left(P_{i}^{t}\right)=\sum_{t=1}^{N} \sum_{i=1}^{n} a_{i}+b_{i} P_{i}^{t}+c_{i}\left(P_{i}^{t}\right)^{2} .
$$

Emission. The amount of emission of $\mathrm{SO}_{2}, \mathrm{CO}_{2}$, and $\mathrm{NO}_{x}$ can be expressed as a quadratic function of the generator's active power output. The total pollutants emission over the dispatch period $[0, N]$ is given by $[23]$

$$
E\left(\mathbf{P}^{0}\right)=\sum_{t=1}^{N} \sum_{i=1}^{n} E_{i}\left(P_{i}^{t}\right)=\sum_{t=1}^{N} \sum_{i=1}^{n} \alpha_{i}+\beta_{i} P_{i}^{t}+\gamma_{i}\left(P_{i}^{t}\right)^{2}
$$

Constraints. (i) Power balance constraint

$$
\sum_{i=1}^{n} P_{i}^{t}=D^{t}+\text { Loss }^{t}, \quad t=1,2, \ldots, N,
$$

where $\operatorname{Loss}^{t}$ is the transmission line loss at time $t$. The Bcoefficient method is one of the most commonly used method by the power utility industry to calculate the network losses. In this method the network losses are expressed as a quadratic function of the generator's power outputs that can be approximated in the following [13]:

$$
\operatorname{Loss}^{t}=\sum_{i=1}^{n} \sum_{j=1}^{n} P_{i}^{t} B_{i j} P_{j}^{t}, \quad t=1,2, \ldots, N .
$$

(ii) Generation limits

$$
P_{i}^{\min } \leq P_{i}^{t} \leq P_{i}^{\max }, \quad i=1,2, \ldots, n, t=1,2, \ldots, N .
$$

(iii) Generating unit ramp rate limits

$$
\begin{array}{r}
-\mathrm{DR}_{i} \cdot T \leq P_{i}^{t+1}-P_{i}^{t} \leq \mathrm{UR}_{i} \cdot T, \quad i=1,2, \ldots, n, \\
t=1,2, \ldots, N-1, \\
-\mathrm{DR}_{i} \cdot T \leq P_{i}^{1}-P_{i}^{N} \leq \mathrm{UR}_{i} \cdot T, \quad i=1,2, \ldots, n .
\end{array}
$$

The Optimization Problem. Aggregating the objectives and constraints, the DEED problem can be mathematically formulated as a nonlinear constrained multi-objective optimization problem which can be converted into a single-objective optimization using the weighting method as follows

$$
\begin{aligned}
& \min _{\mathbf{P}^{0}} H\left(\mathbf{P}^{0}\right)=\alpha C\left(\mathbf{P}^{0}\right)+(1-\alpha) E\left(\mathbf{P}^{0}\right), \\
& \text { subject to } P_{i}^{t} \in \Omega_{\mathrm{DEED}}\left(\mathbf{P}^{0}\right), \quad i=1,2, \ldots, n, \\
& t=1,2, \ldots, N,
\end{aligned}
$$

where the feasible domain $\Omega_{\mathrm{DEED}}$ is defined to be the set of $\left(P_{i}^{t}: i=1,2, \ldots, n, t=1,2, \ldots, N\right)$ satisfying constraints (3)-(7); $\alpha$ is a weighting factor, $\alpha \in[0,1]$. It will be noted that, when $\alpha=1$, the problem (8)-(9) determines the optimal amount of the generated power by minimizing the cost regardless of emission and the DEED problem leads to the DED problem [2]. If $\alpha=0$ then, the DEED problem determines the optimal amount of the generated power by minimizing the emission regardless of cost and the DEED problem leads to the pure dynamic emission dispatch (PDED) [13].

The constraints (3)-(6) are usually used in the conventional DEED problem $[13,16,17]$. Since the demand and constraints are periodic, one may obtain the solution of the conventional DEED problem (i.e., problem (8) under constraints (3)-(6)) over, for example, 24 hours $(N=24$ and $T=1$ ), and then, this solution is implemented not only for the first day but also for all the other week days. Sometimes such an optimal solution is not able to be practically implemented, or, in other words, the solution is not practically feasible. The ramp rate constraint may be violated when the generators are moved from the 24th hour of a day to the first hour of the next day. This problem can be resolved by including the ramp limit on the difference between $P_{i}^{24}$ and $P_{i}^{25}=P_{i}^{1}$. This can be achieved by adding the constraint (7) to the conventional DEED problem (see $[2,20]$ ).

We note that the above DEED problem can be solved over the dispatch period $[m, m+N]$ for any $m \geq 0$ and it can be formulated as

$$
\begin{array}{r}
\min _{\mathbf{P}^{m}} H\left(\mathbf{P}^{m}\right) \\
\text { subject to } P_{i}^{t} \in \Omega_{\mathrm{DEED}}\left(\mathbf{P}^{m}\right), \quad i=1,2, \ldots, n, \\
t=m+1, m+2, \ldots, m+N .
\end{array}
$$

We define the following set of parameters:

$$
\begin{gathered}
\Gamma_{\mathrm{DEED}}=\left\{n, N, \alpha, T, \mathrm{DR}_{i}, \mathrm{UR}_{i}, P_{i}^{\min }, P_{i}^{\max }, a_{i}, b_{i},\right. \\
\left.c_{i}, \alpha_{i}, \beta_{i}, \gamma_{i}, B_{i j}, i, j=1, \ldots, n\right\} .
\end{gathered}
$$

Since the demand $D$ is periodic and $\Gamma_{\mathrm{DEED}}$ do not change over time, then $P^{m+1}=P^{m+N+1}$, and $\Omega_{\mathrm{DEED}}$ satisfies

$$
\begin{aligned}
\Omega_{\mathrm{DEED}}\left(\mathbf{P}^{m+1}\right) & =\Omega_{\mathrm{DEED}}\left(P^{m+2}, \ldots, P^{m+N}, P^{m+N+1}\right) \\
& =\Omega_{\mathrm{DEED}}\left(P^{m+2}, \ldots, P^{m+N}, P^{m+1}\right) \\
& =\Omega_{\mathrm{DEED}}\left(\mathbf{P}^{m}\right)
\end{aligned}
$$

and then $\Omega_{\text {DEED }}$ is shift invariant (see [21]). The shift-invariant property of $\Omega_{\mathrm{DEED}}$ is needed for the application of the MPC approach to the DEED problem.

2.2. Profit-Based Dynamic Economic Emission Dispatch. Now we introduce the PBDEED formulation with the aim to produce electricity with minimum operating cost and sell it with maximum profits and environmental protection by limiting the emission of greenhouse gases into the atmosphere.

The total revenue of the GENCO over the dispatch period $[0, N]$ is given by $[11]$

$$
\mathrm{RV}\left(\mathbf{P}^{0}\right)=\sum_{t=1}^{N} \sum_{i=1}^{n} \mathrm{SP}^{t} \cdot P_{i}^{t}
$$

where $\mathrm{SP}^{t}$ is the forecasted energy price at time $t$. 

$[11,20]$

The total profit over the dispatch period $[0, N]$ is given by

$$
\operatorname{PF}\left(\mathbf{P}^{0}\right)=\operatorname{RV}\left(\mathbf{P}^{0}\right)-C\left(\mathbf{P}^{0}\right) .
$$

Let us define a function $G(\cdot)=-\mathrm{PF}(\cdot)$ which measures the profit attained by the conversion of the energy available in fossil fuels into electric energy.

The objective of the PBDEED is to simultaneously minimize the emission and maximize the profit satisfying a set of constraints. The PBDEED can be mathematically stated as follows:

$$
\begin{aligned}
& \min _{\mathbf{P}^{0}} \alpha G\left(\mathbf{P}^{0}\right)+(1-\alpha) E\left(\mathbf{P}^{0}\right) \\
& \text { subject to } P_{i}^{t} \in \Omega_{\mathrm{PBDEED}}\left(\mathbf{P}^{0}\right), \\
& i=1,2, \ldots, n, \\
& t=1,2, \ldots, N,
\end{aligned}
$$

where the feasible domain $\Omega_{\mathrm{PBDEED}}$ is defined to be the set of $\left(P_{i}^{t}: i=1,2, \ldots, n, t=1,2, \ldots, N\right)$ satisfying the following constraints:

$$
\begin{gathered}
\sum_{i=1}^{n} P_{i}^{t} \leq D^{t}+\operatorname{Loss}^{t}, \quad t=1,2, \ldots, N \\
P_{i}^{\min } \leq P_{i}^{t} \leq P_{i}^{\max }, \quad i=1,2, \ldots, n, t=1,2, \ldots, N \\
-\mathrm{DR}_{i} \cdot T \leq P_{i}^{t+1}-P_{i}^{t} \leq \mathrm{UR}_{i} \cdot T, \quad i=1,2, \ldots, n, \\
t=1,2, \ldots, N-1, \\
-\mathrm{DR}_{i} \cdot T \leq P_{i}^{1}-P_{i}^{N} \leq \mathrm{UR}_{i} \cdot T, \quad i=1,2, \ldots, n
\end{gathered}
$$

It will be noted that, when $\alpha=1$, the problem (15) determines the optimal amount of the generated power by maximizing the profit regardless of emission and the PBDEED problem leads to the PBDED problem [11]. If $\alpha=0$, then the PBDEED problem determines the optimal amount of the generated power by minimizing the emission regardless of profit [24]. Of course this case is not useful for GENCOs.

Constraint (16) means that under the deregulated environment GENCO is not obliged to meet all demands and energy lost due to transmission lines but may sell its energy at less than the system's forecasted demand plus power loss. Similar to Section 2.1, one can show that the set $\Omega_{\text {PBDEED }}$ is shift invariant.

In the next section we propose an MPC approach for the periodic implementation of the optimal solutions of the DEED problem. MPC obtains a feedback control which is constructed by solving a finite horizon optimal control problem at each sampling instant using the current state of the plant as the initial state for the optimization and applying only "the first part" of the optimal control [25].

\section{MPC Approach to DEED}

In this section, we introduce an MPC approach proposed in $[2,20]$ for the optimal dynamic dispatch problem with emission and transmission line losses. We first show the application of MPC to the DEED problem and then show that this MPC version can also be applied to the PBDEED problem. We introduce the control variables $u_{i}^{t}$ as $[8,9]$

$$
u_{i}^{t}=\frac{P_{i}^{t+1}-P_{i}^{t}}{T}, \quad i=1,2, \ldots, n, t=1,2, \ldots, N-1,
$$

where $u_{i}^{t}$ is the ramping action of unit $i$ at time $t$. This equation actually defines coordinate transformation between the variables $\left\{P_{i}^{t}: i=1,2, \ldots, n, t=1,2, \ldots, N\right\}$ and the variables $\left\{u_{i}^{t}: i=1,2, \ldots, n, t=1,2, \ldots, N-1\right\}$. It is obvious that the inverse coordinate transformation is given by

$$
P_{i}^{t}=P_{i}^{1}+\sum_{j=1}^{t-1} T u_{i}^{j}, \quad t=2,3, \ldots, N
$$

The optimal solution of the DEED problem is implemented repeatedly at instants which equal to multiples of $N$. To introduce the MPC approach, let us consider the DEED problem starting at an arbitrary instant $t=m$ and over a dispatch interval $[m, m+N)$. Then the optimization variables are changed into $\left\{P_{i}^{m+1}, P_{i}^{m+2}, \ldots, P_{i}^{m+N}, i=1,2, \ldots, n\right\}$. By the transformation defined in (21), the optimization variables $\left\{P_{i}^{m+1}, P_{i}^{m+2}, \ldots, P_{i}^{m+N}, i=1,2, \ldots, n\right\}$ are transformed into $\left\{P_{i}^{m+1}, u_{i}^{m+1}, \ldots, u_{i}^{m+N-1}, i=1,2, \ldots, n\right\}$.

In the MPC method, a finite-horizon optimal control problem is repeatedly solved and the input is applied to the system based on the obtained optimal open-loop control. In our case, the horizon is chosen to be $N$. Instead of solving the DEED problem with $n N$ number of variables $\left\{P_{i}^{m+1}\right.$, $\left.u_{i}^{m+1}, \ldots, u_{i}^{m+N-1}, i=1,2, \ldots, n\right\}$, the MPC algorithm solves the following problem which has only $n(N-1)$ number of variables $\left\{u_{i}^{m+1}, \ldots, u_{i}^{m+N-1}, i=1,2, \ldots, n\right\}$.

Problem MPCDEED $P_{P^{m+1}}(u,[m, m+N))$. Given $\Gamma_{\mathrm{DEED}}, D$ and $P^{m+1}$, let

$$
\begin{array}{r}
P_{i}^{1}:=P_{i}^{m+1}, \quad u_{i}^{j}:=u_{i}^{m+j}, \quad D^{t}:=D^{m+t}, \\
i=1,2, \ldots, n, t=1,2, \ldots, N, j=1,2, \ldots, N-1,
\end{array}
$$

and solves the following minimization problem:

$$
\min _{\mathbf{U}} \sum_{t=1}^{N} \sum_{i=1}^{n} \alpha C_{i}\left(P_{i}^{1}+\sum_{s=1}^{t-1} T u_{i}^{s}\right)+(1-\alpha) E_{i}\left(P_{i}^{1}+\sum_{s=1}^{t-1} T u_{i}^{s}\right)
$$

subject to $u_{i}^{t} \in \Omega_{D}\left(P^{1}, U\right), \quad i=1,2, \ldots, n$,

$$
t=1,2, \ldots, N-1 \text {, }
$$




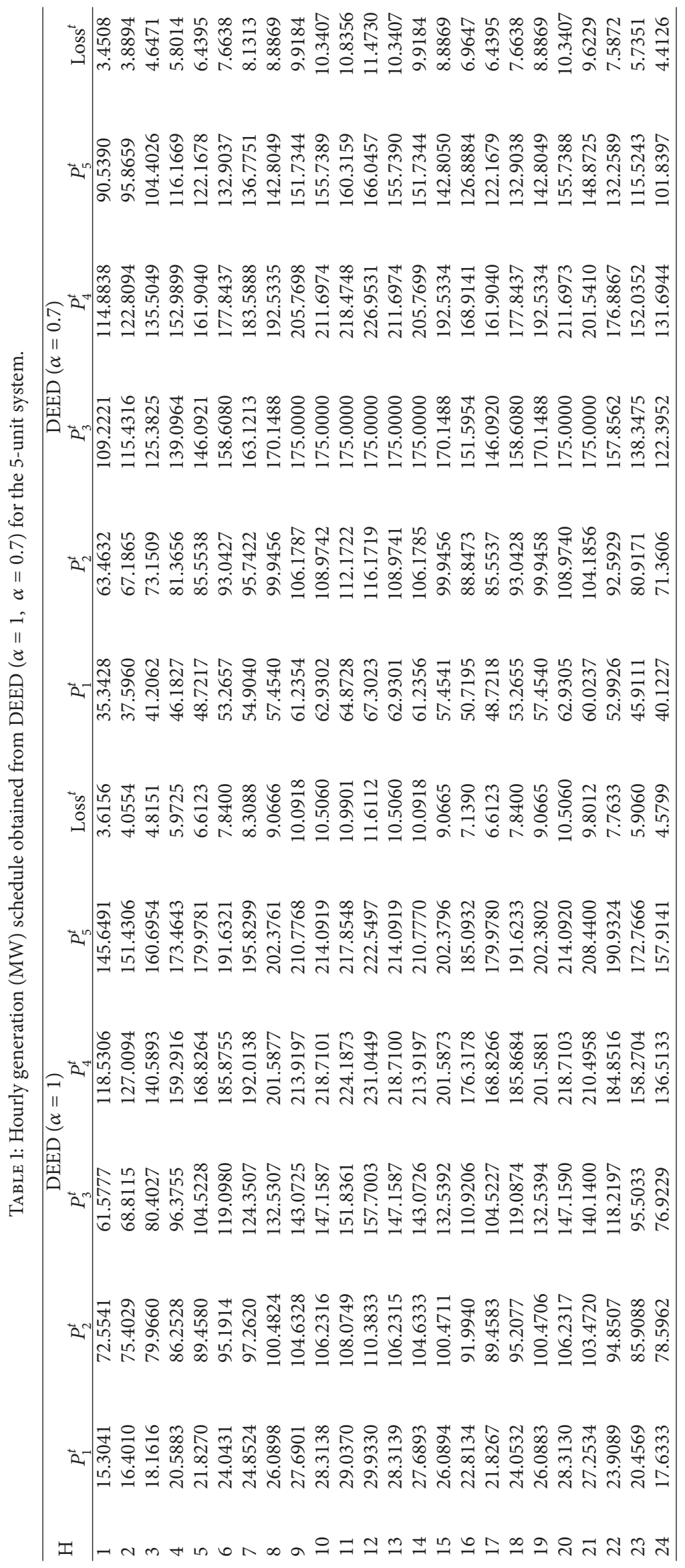




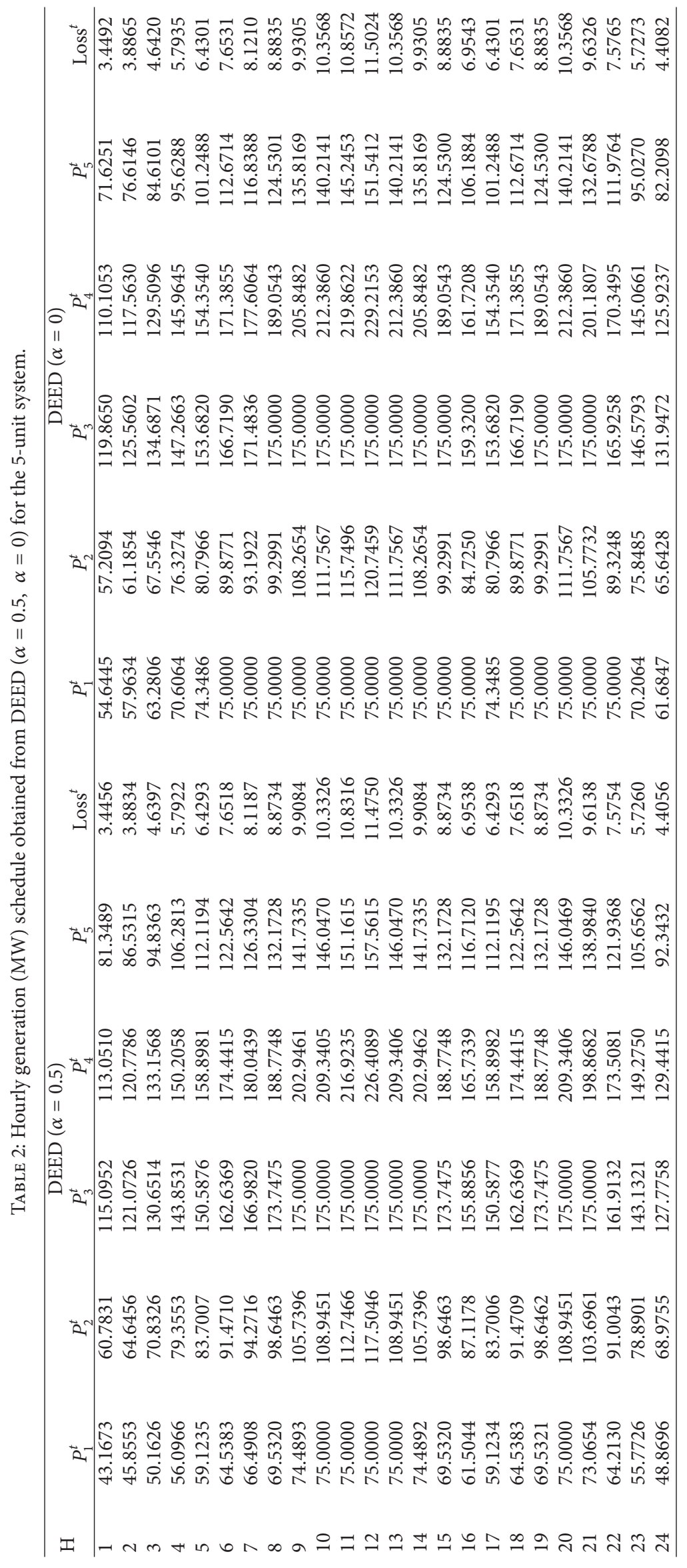


TABLE 3: The optimal cost and emission obtained from DEED with different value of $\alpha$ for the 5-unit system.

\begin{tabular}{lcc}
\hline Value of $\alpha$ & Cost (\$) & Emission (lb) \\
\hline$\alpha=1$ & 40,121 & 20,363 \\
$\alpha=0.9$ & 40,215 & 18,100 \\
$\alpha=0.8$ & 40,343 & 17,340 \\
$\alpha=0.7$ & 40,465 & 16,966 \\
$\alpha=0.6$ & 40,578 & 16,752 \\
$\alpha=0.5$ & 40,670 & 16,638 \\
$\alpha=0.4$ & 40,731 & 16,587 \\
$\alpha=0.3$ & 40,775 & 16,563 \\
$\alpha=0.2$ & 40,808 & 16,552 \\
$\alpha=0.1$ & 40,832 & 16,547 \\
$\alpha=0$ & 40,851 & 16,546 \\
\hline
\end{tabular}

where the feasible domain $\Omega_{D}\left(P^{1}, U\right)$ is defined to be the set of $\left\{P_{i}^{1}, u_{i}^{t}: i=1,2, \ldots, n, t=1,2, \ldots, N-1\right\}$ satisfying

$$
\begin{aligned}
& \sum_{i=1}^{n}\left(P_{i}^{1}+\sum_{s=1}^{t-1} T u_{i}^{s}\right) \\
& =D^{t}+\sum_{i=1}^{n} \sum_{j=1}^{n}\left(P_{i}^{1}+\sum_{s=1}^{t-1} T u_{i}^{s}\right) B_{i j}\left(P_{j}^{1}+\sum_{s=1}^{t-1} T u_{j}^{s}\right), \\
& t=1,2, \ldots, N, \\
& P_{i}^{\min } \leq P_{i}^{1}+\sum_{s=1}^{t-1} T u_{i}^{s} \\
& \leq P_{i}^{\max }, \quad i=1,2, \ldots, n, t=1,2, \ldots, N, \\
& -\mathrm{DR}_{i} \leq u_{i}^{t} \quad \\
& \leq \mathrm{UR}_{i}, \quad i=1,2, \ldots, n, t=1,2, \ldots, N-1 .
\end{aligned}
$$

The notation $\operatorname{MPCDEED}_{P^{m+1}}(u,[m, m+N))$ denotes that the optimization problem is solved over the interval $[m, m+$ $N)$ with variables $u_{i}^{t}$ and for known inputs $P_{i}^{m+1}, i=1$, $2, \ldots, n, \quad t=m+1, \ldots, m+N-1$.

In order to make the MPCDEED problem solvable, the following hypothesis is needed as in $[2,5,8]$.

Feasibility Hypothesis. After the change of variables in (22) over any dispatch interval $[m, m+N)$ with $m \geq 0$, the set $\Omega_{D}\left(P^{1}, U\right)$ is not empty. This hypothesis ensures the solvability of the problem $\operatorname{MPCDEED}_{P^{m+1}}(u,[m, m+N))$. Denote the optimal solution of MPCDEED for a given initial generation $\bar{P}^{m+1}$ by $\overline{\mathbf{u}}^{m}\left(\bar{P}^{m+1}\right)=\left\{\bar{u}_{i}^{m+j}\left(\bar{P}^{m+1}\right), i=1,2, \ldots, n, j=\right.$ $1,2, \ldots, N-1\}$. In the MPC method, the optimal solution $\overline{\mathbf{u}}^{m}$ is applied only in the first sampling period $[m, m+$ 1); that is, $\bar{u}_{i}^{m+1}\left(\bar{P}^{m+1}\right)$ is applied to the state $\bar{P}_{i}^{m+1}$. Since $\overline{\mathbf{u}}^{m}\left(\bar{P}^{m+1}\right)$ depends on the current state $\bar{P}^{m+1}$, in this way a feedback control can be obtained. We define the MPC feedback controller by $v_{i}^{m}:=\bar{u}_{i}^{m+1}$. The closed-loop solution
$\bar{P}_{i}^{m+2}$ given by $\bar{P}_{i}^{m+2}=\bar{P}_{i}^{m+1}+T v_{i}^{m}\left(\bar{P}^{m+1}\right)$ is actually executed over the time period $[m+1, m+2)$. The idea of the MPC can be formulated into the following MPC algorithm.

MPC Algorithm Initialization. Input the initial status $\bar{P}^{1} \triangleq$ $P^{1}=\left(P_{1}^{1}, P_{2}^{1}, \ldots, P_{n}^{1}\right)$ and let $m=0$.

(1) Compute the open-loop optimal solution $\overline{\mathbf{u}}^{m}$ to the problem $\operatorname{MPCDEED}_{\bar{P}^{m+1}}(u,[m, m+N))$.

(2) The (closed-loop) MPC controller $v_{i}^{m}$ is applied to the plant in the sampling interval $[m, m+1)$ to obtain the closed-loop MPC solution

$$
\bar{P}_{i}^{m+2}=\bar{P}_{i}^{m+1}+T v_{i}^{m}\left(\bar{P}^{m+1}\right)
$$

over the period $[m+1, m+2)$.

(3) Let $m:=m+1$ and go to step (1).

Theorem 1 (see [2]). Suppose the Feasibility Hypothesis holds, $P^{*}$ is the globally optimal solution of the DEED problem, and the initial power output $P^{1}$ at time $t=1$ satisfies $P_{i}^{1} \in \Omega_{D E E D}$, then the MPC algorithm converges to $P^{*}$.

This theorem tells that the solutions of the MPC algorithm converge to the optimal solution of the DEED problem. Now we consider the inherent robustness properties of the MPC algorithm. The uncertainties in energy demand, price, and reserve demand for the PBDED problem are discussed by fuzzy optimization in [11]. However, no theoretical result is given. First, we suppose that the disturbances happen in the execution of the controller. That is, the disturbance happens only in step (2) of the MPC algorithm so that when the control $v_{i}^{m}$ is applied to the plant in the sampling interval $[m, m+1)$, the system actually executes

$$
\bar{P}_{i}^{m+2}=\bar{P}_{i}^{m+1}+T v_{i}^{m}\left(\bar{P}^{m+1}\right)+T w_{i}^{m+1}
$$

over the period $[m+1, m+2)$, where $w_{i}^{m+1}$ is the disturbance. We assume that the disturbances satisfy the following bound:

$$
\left\|w_{i}^{m+1}\right\|<e, \quad e>0, \quad i=1,2, \ldots, n, m \geq 0 .
$$

Theorem 2 (see [2]). Suppose the Feasibility Hypothesis holds, $P^{*}$ is the globally optimal solution of the DEED problem, the norm of the gradient of the function $H$ of DEED problem has the upper bound $L$ on $\Omega_{D E E D}$, $\epsilon$ is a small enough positive constant, $c$ is a positive constant which is less than $\epsilon,(26)$ is executed in step (2) of the MPC algorithm instead of (25), and the constant disturbance $w_{i}^{k}$ satisfies (27) where e is small enough so that $e<\min \{c / L,(\epsilon-c) / L\}$, then there exists an integer $N_{0}$ such that for any $k>N_{0}$, the optimal MPC solution $\bar{P}^{k+1}$ of the kth loop in the MPC algorithm belongs to the domain $\bar{\Omega}:=\left\{P:\left\|P-P^{*}\right\|<c\right\}$.

Theorem 2 shows that the MPC algorithm is robust against certain disturbances in the execution of the optimal controller. It may happen that there is disturbance or uncertainty in the forecasted demand; that is, the demand $D^{k}$ is 
TABLE 4: The results of PBDEED $(\alpha=1)$ for the three price profiles.

\begin{tabular}{|c|c|c|c|c|c|c|c|c|c|}
\hline $\mathrm{H}$ & $\begin{array}{c}\text { Price-I } \\
(\$)\end{array}$ & $\begin{array}{c}P L^{t} \\
(\mathrm{MW})\end{array}$ & $\begin{array}{l}\text { Loss }^{t} \\
(\mathrm{MW})\end{array}$ & $\begin{array}{c}\text { Price-II } \\
(\$)\end{array}$ & $\begin{array}{c}P L^{t} \\
(\mathrm{MW})\end{array}$ & $\begin{array}{l}\text { Loss }^{t} \\
(\mathrm{MW})\end{array}$ & $\begin{array}{c}\text { Price-III } \\
(\$)\end{array}$ & $\begin{array}{c}P L^{t} \\
(\mathrm{MW})\end{array}$ & $\begin{array}{l}\text { Loss }^{t} \\
(\mathrm{MW})\end{array}$ \\
\hline 1 & 10.31 & 561.02 & 4.25 & 11.46 & 740.1 & 7.5 & 13.75 & 955 & 11.76 \\
\hline 2 & 10.80 & 621.59 & 5.39 & 12.00 & 873.8 & 10.0 & 14.40 & 942 & 11.51 \\
\hline 3 & 11.56 & 745.91 & 7.50 & 12.84 & 935 & 11.3 & 15.41 & 935 & 11.40 \\
\hline 4 & 9.49 & 482.50 & 3.01 & 10.54 & 615.2 & 5.3 & 12.65 & 930 & 11.21 \\
\hline 5 & 10.89 & 625.04 & 5.46 & 12.10 & 866.8 & 9.8 & 14.52 & 935 & 11.37 \\
\hline 6 & 11.86 & 835.62 & 9.34 & 13.18 & 963 & 11.9 & 15.82 & 963 & 12.00 \\
\hline 7 & 12.42 & 989 & 12.45 & 13.80 & 989 & 12.5 & 16.56 & 989 & 12.58 \\
\hline 8 & 11.21 & 803.18 & 8.62 & 12.46 & 1008 & 12.9 & 14.95 & 1023 & 13.28 \\
\hline 9 & 13.25 & 1124.56 & 15.45 & 14.72 & 1126 & 15.7 & 17.66 & 1126 & 15.78 \\
\hline 10 & 13.45 & 1150 & 16.17 & 14.94 & 1150 & 16.2 & 17.93 & 1150 & 16.36 \\
\hline 11 & 13.37 & 1201 & 17.41 & 14.86 & 1201 & 17.5 & 17.83 & 1201 & 17.60 \\
\hline 12 & 13.91 & 1235 & 18.29 & 15.46 & 1235 & 18.4 & 18.55 & 1235 & 18.50 \\
\hline 13 & 11.74 & 805.70 & 8.75 & 13.04 & 1190 & 17.1 & 15.65 & 1190 & 17.23 \\
\hline 14 & 12.42 & 996.35 & 12.61 & 13.80 & 1251 & 18.7 & 16.56 & 1251 & 18.82 \\
\hline 15 & 10.98 & 645.56 & 5.86 & 12.20 & 932.2 & 11.2 & 14.64 & 1263 & 19.04 \\
\hline 16 & 10.19 & 545.29 & 3.98 & 11.32 & 726.9 & 7.2 & 13.58 & 1250 & 18.66 \\
\hline 17 & 11.25 & 698.05 & 6.76 & 12.50 & 999.9 & 12.7 & 15.00 & 1221 & 17.98 \\
\hline 18 & 12.26 & 895.99 & 10.14 & 13.62 & 1202 & 17.2 & 16.34 & 1202 & 17.56 \\
\hline 19 & 9.79 & 541.62 & 3.94 & 10.88 & 697.4 & 6.8 & 13.06 & 1159 & 16.37 \\
\hline 20 & 11.03 & 655.09 & 6.03 & 12.26 & 943.6 & 11.5 & 14.71 & 1092 & 14.87 \\
\hline 21 & 11.20 & 686.22 & 6.56 & 12.44 & 1002.2 & 12.7 & 14.93 & 1023 & 13.28 \\
\hline 22 & 12.22 & 929.72 & 11.23 & 13.58 & 984 & 12.4 & 16.30 & 984 & 12.47 \\
\hline 23 & 11.43 & 732.97 & 7.41 & 12.70 & 975 & 12.1 & 15.24 & 975 & 12.24 \\
\hline 24 & 10.28 & 556.53 & 4.17 & 11.42 & 730.59 & 7.4 & 13.70 & 960 & 11.87 \\
\hline \multicolumn{4}{|c|}{ Profit $=831.24(\$)$} & \multicolumn{3}{|c|}{ Profit $=29080(\$)$} & \multicolumn{3}{|c|}{ Profit $=94922.18(\$)$} \\
\hline
\end{tabular}

disturbed so that the actual demand is $\widetilde{D}^{k}$. It has been shown in [2] that the demand disturbances or uncertainties can also be written in the form of (26) and the result of Theorem 2 is applicable when $\left\|\widetilde{D}^{k}-D^{k}\right\|$ is small enough.

Theorems 1 and 2 are based on the assumption that the objective function of the DEED problem is strictly convex and differentiable over the set $\Omega_{\text {DEED }}$ which is bounded. Since both the fuel cost and emission functions are assumed to be quadratic, then all the objective functions of the DEED and PBDEED problems are strictly convex and differentiable over their feasible constraint sets. Also since the demand and energy price and all constraints are assumed to be periodic, then all feasible constraint sets, $\Omega_{\text {DEED }}$ and $\Omega_{\text {PBDEED }}$, are shift invariant. Therefore, Theorems 1 and 2 are valid for both the DEED and PBDEED problems.

\section{Simulation Results}

In this section we present two test systems consisting of five units and six units. The first system is used to show that the MPC algorithm converges to the optimal solutions of the DEED problem. The inherent robustness properties of the model predictive control (IRP-MPC) algorithm are also shown. In the second test system, we show the converge and robustness properties of the proposed MPC algorithm to the
PBDEED problem. The load demand and energy price are assumed to be periodic over a dispatch period of one day and the sampling period is chosen to be one hour. The optimization problem is solved by sequential quadratic programming. All computations were carried out by the MATLAB program. In particular, the optimal control sequence is computed by the fmincon code of the MATLAB Optimization Toolbox.

4.1. Five-Unit Test System. This example presents an application of MPC to the DEED problem consisting of five units. The technical data of the units are taken from [16]. First, we present the optimal solutions of the DEED problems for different values of the weighting factor $\alpha$ and then show that the solutions of the MPC converge to the optimal solutions of the DEED problem. The optimal solutions of the DEED problem with different values of $\alpha$ are given in Tables 1 and 2 . Table 1 shows the hourly power schedule and transmission loss obtained from the DEED problem with $\alpha=1$ and $\alpha=0.7$. Table 2 shows the hourly power schedule and transmission loss obtained from the DEED problem with $\alpha=0.5$ and $\alpha=0$. The optimal cost and emission obtained from the DEED problem with different values of $\alpha$ are given in Table 3. It is seen from Table 3 that the cost is $\$ 40,121$ under $\operatorname{DEED}(\alpha=1)$, but it increases to $\$ 40,851$ under DEED $(\alpha=$ $0)$ and emission obtained from DEED $(\alpha=1)$ is $20,363 \mathrm{lb}$ 
TABLE 5: The results of PBDEED for price-II with different values of $\alpha$.

\begin{tabular}{|c|c|c|c|c|c|c|c|c|}
\hline \multirow[b]{2}{*}{$\mathrm{H}$} & \multicolumn{2}{|c|}{$\alpha=0.2$} & \multicolumn{2}{|c|}{$\alpha=0.5$} & \multicolumn{2}{|c|}{$\alpha=0.7$} & \multicolumn{2}{|c|}{$\alpha=1$} \\
\hline & $\begin{array}{c}P L^{t} \\
(\mathrm{MW})\end{array}$ & $\begin{array}{l}\text { Loss }^{t} \\
(\mathrm{MW})\end{array}$ & $\begin{array}{c}P L^{t} \\
(\mathrm{MW})\end{array}$ & $\begin{array}{l}\text { Loss }^{t} \\
(\mathrm{MW})\end{array}$ & $\begin{array}{c}P L^{t} \\
(\mathrm{MW})\end{array}$ & $\begin{array}{l}\operatorname{Loss}^{t} \\
(\mathrm{MW})\end{array}$ & $\begin{array}{c}P L^{t} \\
(\mathrm{MW})\end{array}$ & $\begin{array}{l}\text { Loss }^{t} \\
(\mathrm{MW})\end{array}$ \\
\hline 1 & 385.3 & 1.5 & 516.3 & 3.2 & 613 & 4.9 & 740.1 & 7.5 \\
\hline 2 & 400.5 & 1.6 & 589 & 4.1 & 717.4 & 6.5 & 873.9 & 10.1 \\
\hline 3 & 438.6 & 1.9 & 746 & 6.4 & 913.1 & 9.9 & 935 & 11.3 \\
\hline 4 & 378.5 & 1.5 & 438.8 & 2.2 & 512.9 & 3.3 & 615.2 & 5.3 \\
\hline 5 & 403.7 & 1.6 & 606 & 4.4 & 738.2 & 6.8 & 866.8 & 9.8 \\
\hline 6 & 463.2 & 2.1 & 821.4 & 7.6 & 963 & 10.9 & 963 & 11.9 \\
\hline 7 & 516.5 & 2.7 & 958.6 & 10 & 989 & 11.4 & 989 & 12.5 \\
\hline 8 & 415.6 & 1.7 & 742.6 & 6.2 & 827.1 & 8.3 & 1008 & 12.9 \\
\hline 9 & 610.2 & 3.7 & 1081.2 & 12.7 & 1126 & 14.4 & 1126 & 15.7 \\
\hline 10 & 633.2 & 4.0 & 1150 & 14.2 & 1150 & 15.0 & 1150 & 16.2 \\
\hline 11 & 624.8 & 3.9 & 1173.5 & 14.8 & 1201 & 16.2 & 1201 & 17.5 \\
\hline 12 & 687.5 & 4.7 & 1235 & 16.3 & 1235 & 17.1 & 1235 & 18.4 \\
\hline 13 & 451.7 & 2.0 & 790.4 & 7.1 & 973.7 & 11.1 & 1190 & 17.1 \\
\hline 14 & 516.5 & 2.7 & 958.6 & 10 & 1187.7 & 15.9 & 1251 & 18.7 \\
\hline 15 & 406.9 & 1.7 & 624.2 & 4.6 & 763.7 & 7.2 & 932.2 & 11.2 \\
\hline 16 & 382.7 & 1.5 & 500.3 & 3.0 & 592 & 4.6 & 726.9 & 7.2 \\
\hline 17 & 417.4 & 1.7 & 678.6 & 5.4 & 827.4 & 8.4 & 999.7 & 12.7 \\
\hline 18 & 501.0 & 2.5 & 913.7 & 9.2 & 1075.7 & 13.1 & 1202 & 17.2 \\
\hline 19 & 378.5 & 1.5 & 465.6 & 2.5 & 567.9 & 4.2 & 697.4 & 6.8 \\
\hline 20 & 408.9 & 1.7 & 635.1 & 4.8 & 777.6 & 7.5 & 943.6 & 11.5 \\
\hline 21 & 414.8 & 1.7 & 667.7 & 5.2 & 819.2 & 8.2 & 1002.2 & 12.7 \\
\hline 22 & 497.6 & 2.5 & 910 & 9.1 & 984 & 11.3 & 984 & 12.4 \\
\hline 23 & 429.5 & 1.8 & 714.9 & 5.9 & 879.2 & 9.3 & 975 & 12.2 \\
\hline 24 & 384.5 & 1.5 & 511.7 & 3.1 & 605.4 & 4.8 & 0.730 .6 & 7.4 \\
\hline Profit (\$) & \multicolumn{2}{|c|}{3,225} & \multicolumn{2}{|c|}{23,505} & \multicolumn{2}{|c|}{27,596} & \multicolumn{2}{|c|}{29,080} \\
\hline
\end{tabular}

but decreases to $16,546 \mathrm{lb}$ under DEED $(\alpha=0)$. Figure 1 shows the effect of the transmission loss on the outputs of the power units. We can see that neglecting transmission loss would often introduce inaccuracy into the resulting dispatch.

Turning now to our goal of this section, we apply MPC strategy. The initial $P_{i}^{1}$ is chosen such that $\sum_{i=1}^{n} P_{i}^{1}=D^{1}+$ $\sum_{i=1}^{n} \sum_{j=1}^{n} P_{i}^{1} B_{i j} P_{j}^{1}$. The proposed MPC strategy is implemented over 48 hours. Figures 2, 3, 4, 5, and 6 show that the MPC closed-loop solutions asymptotically approach the optimal solutions of the DEED problem for different values of $\alpha$.

Now we show the IRP-MPC algorithm against two sources of disturbances.

(1) Disturbance arises from the execution of the optimal controller. In this case, we execute (26) with disturbance $w_{i}^{m}$ generated by

$$
w_{i}^{m}=-\varepsilon_{i}+2 \varepsilon_{i} r(m),
$$

where the parameters $r(m)$ 's are uniformly distributed random numbers on $[0,1]$. Denote $\varepsilon=\left(\varepsilon_{1}, \varepsilon_{2}, \ldots, \varepsilon_{5}\right)$. We choose two different bounds of disturbances:

$$
\begin{aligned}
& \text { IRP-MPC-(I): } \varepsilon=(3,3,2,3,2), \\
& \text { IRP-MPC-(II): } \varepsilon=(6,6,4,6,4) .
\end{aligned}
$$

(2) Disturbance arises from the forecasted demand. In this case, the actual demand $\widetilde{D}^{t}$ can be defined as

$$
\widetilde{D}^{t}= \begin{cases}D^{t}, & \text { if } t=1, \\ D^{t}+q(1-2 r(t)) D^{t} & \text { if } t=2,3, \ldots, N,\end{cases}
$$

where $q(1-2 r)$ is the relative change between the actual $\widetilde{D}^{t}$ demand and the forecasted demand $D^{t}$. Here, we assume the demand is disturbed with $5 \%$ and $10 \%$ of the nominal one; then we have the following cases:

$$
\begin{aligned}
& \text { IRP-MPC-(III): } q=0.05, \\
& \text { IRP-MPC-(IV): } q=0.1 .
\end{aligned}
$$

In these cases the initial $P_{i}^{1}, i=1,2 \ldots, 5$ for the MPC are chosen as the optimal solution of the DEED problem at $t=1$; that is, $P_{i}^{1}=\bar{P}_{i}^{1}$, and the weighting factor $\alpha$ is chosen $\alpha=0.5$. From Figures 7, 8, 9, and 10, we can see that, although the disturbance increased, the MPC still keeps the disturbed system in the neighborhood of the optimal solution of the DEED problem. From these cases we observe that the size of this neighborhood depends on the bound of the disturbance.

4.2. Six-Unit Test System. This system consists of six units for the application of MPC to the PBDEED problem. 


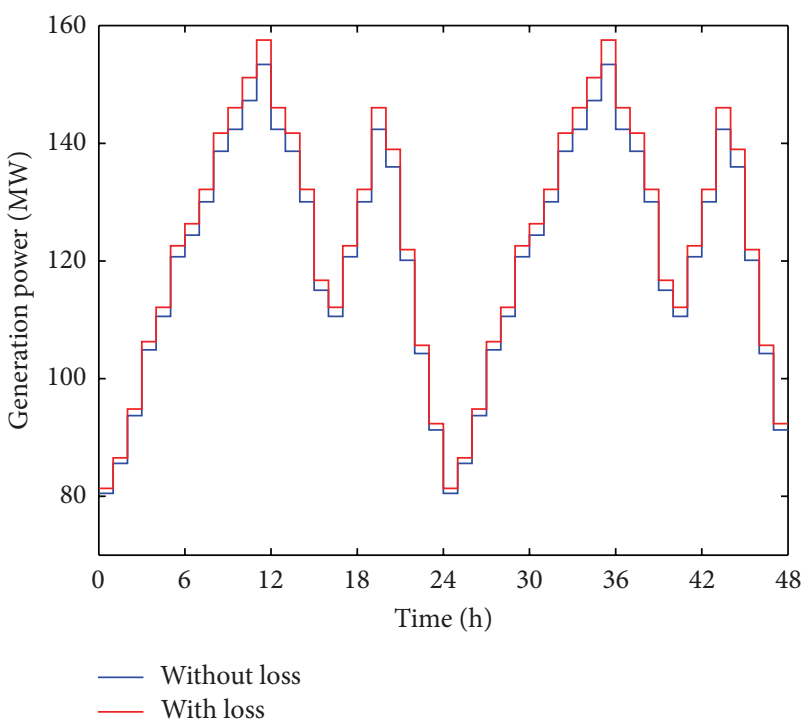

Figure 1: The generation output of unit 5 under DEED $(\alpha=0.5)$ from the five-unit system.

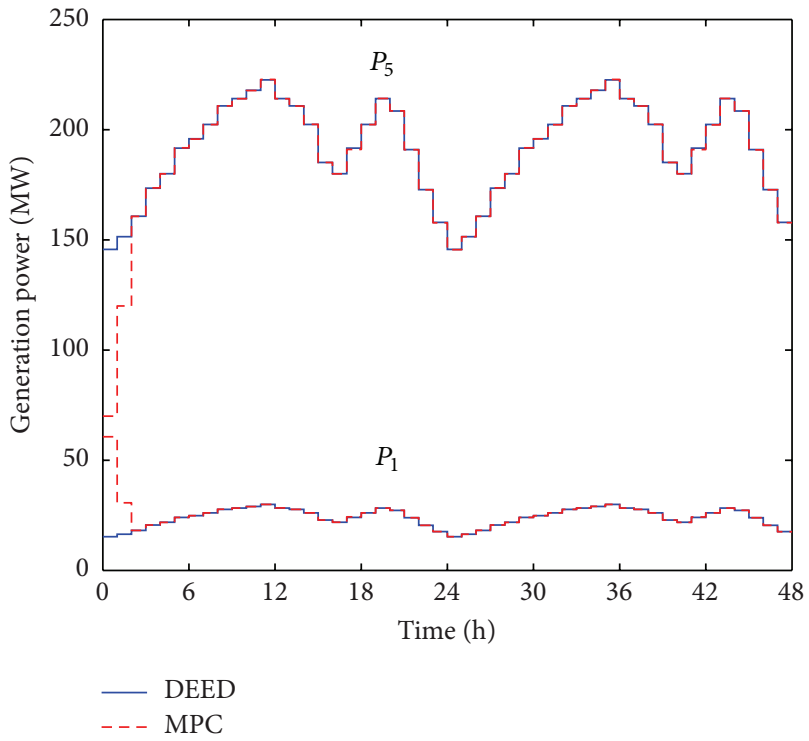

FIGURE 2: Convergence of the closed-loop MPC solutions to those of DEED $(\alpha=1)$ for the five-unit system.

The technical data of the units are taken from [20]. The transmission loss formula coefficients are given in the appendix. The initial $P_{i}^{1}$ is chosen such that $\sum_{i=1}^{n} P_{i}^{1} \leq D^{1}+\sum_{i=1}^{n}$ $\sum_{j=1}^{n} P_{i}^{1} B_{i j} P_{j}^{1}$. The result of the optimization is dependent on the energy price data. Indeed, minor changes in the energy price may give a significant change in the power generation of the units as well as the profit. We consider different energy price profiles which are given in Table 4.

The effect of the energy price over the time horizon on the total power which produced by the committed units for the PBDEED problem at $\alpha=1$ is shown in Table 4 . Now we define $P L^{t}=\sum_{i=1}^{n} P_{i}^{t}-$ Loss $^{t}$. We can see from Table 4 that,

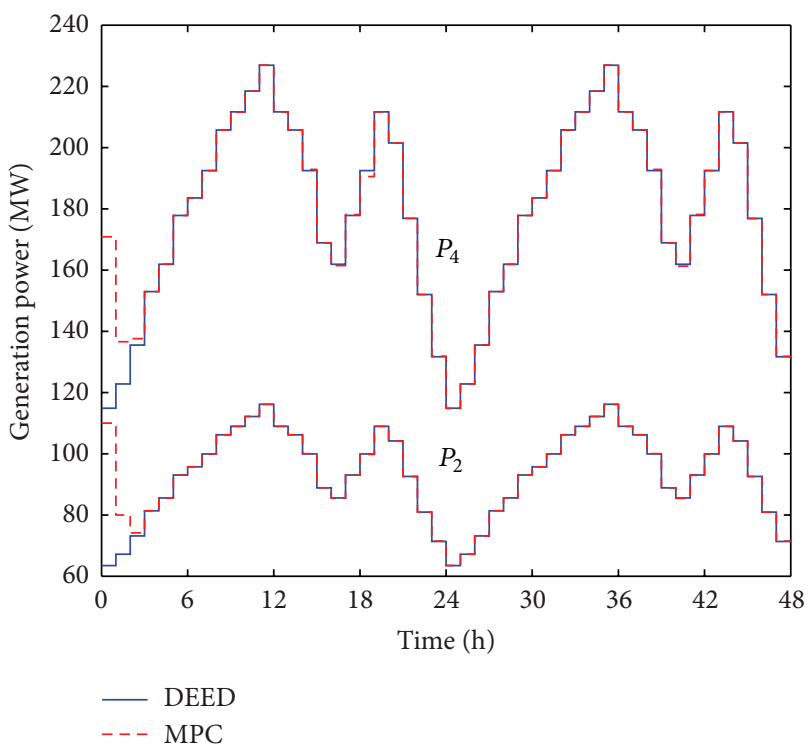

FIGURE 3: Convergence of the closed-loop MPC solutions to those of DEED $(\alpha=0.7)$ for the five-unit system.

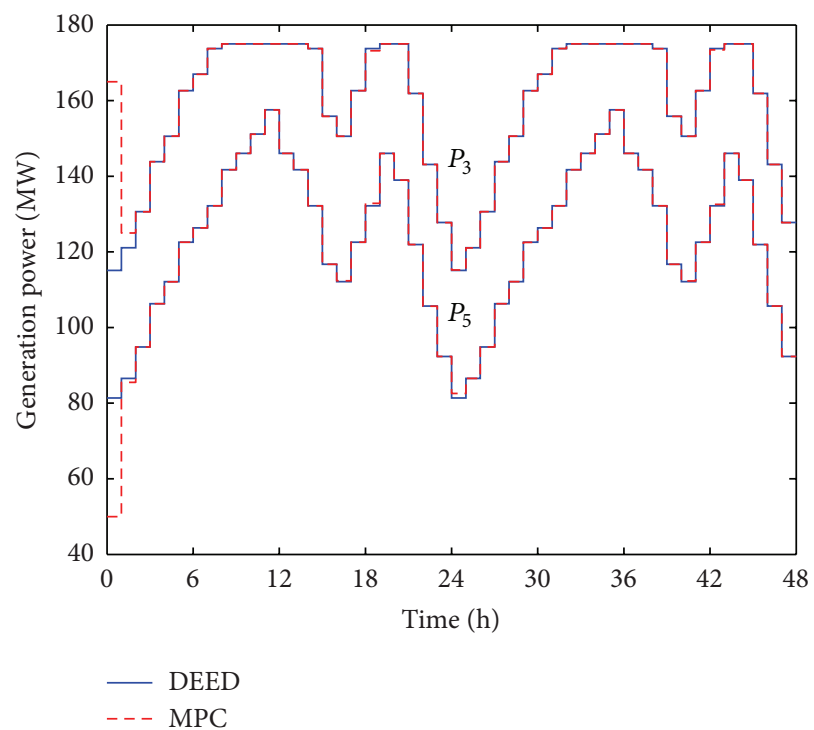

FIGURE 4: Convergence of the closed-loop MPC solutions to those of DEED $(\alpha=0.5)$ for the five-unit system.

for price-I and price-II, $P L^{t}<D^{t}$ in some $t$ and $P L^{t}=D^{t}$ in some other $t$ which are shown by bold face. It means that GENCO chooses to supply power less than the demand plus power loss in some hours of the day and equal to the demand plus power loss in other hours of the day when this satisfies maximum profit. For price-III, the optimal solution of the PBDEED satisfies $P L^{t}=D^{t}$ for all $t$. In this case, GENCO will supply power to satisfy the demand plus power loss over the whole day since this will maximize its profit. We also note that the profit increases according to the increase of the energy prices. According to the PBDEED formulation, GENCO will 


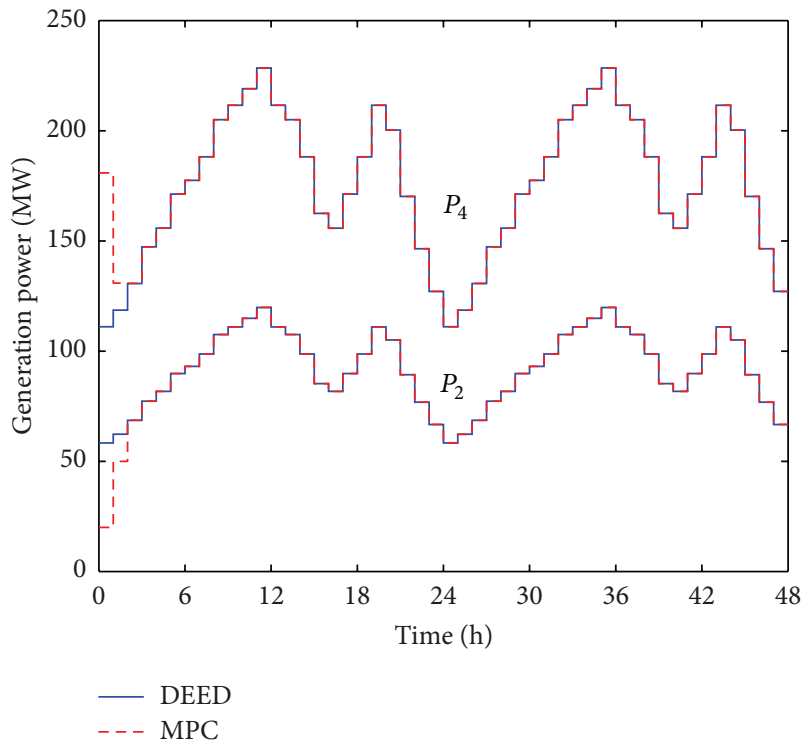

Figure 5: Convergence of the closed-loop MPC solutions to those of DEED $(\alpha=0.2)$ for the five-unit system.

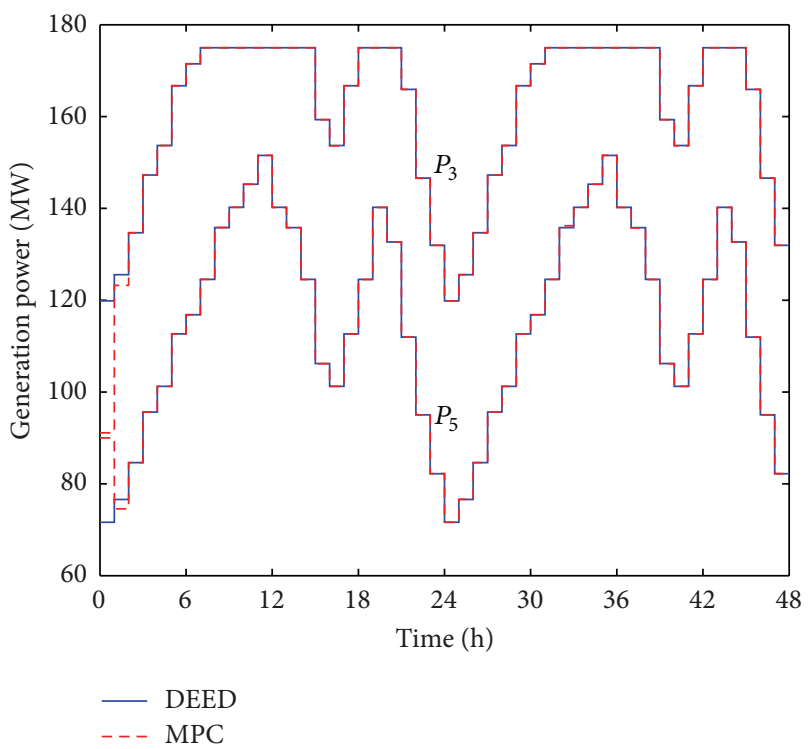

FIGURE 6: Convergence of the closed-loop MPC solutions to those of DEED $(\alpha=0)$ for the five-unit system.

sell all produced power and the consumer is the one who will bear the energy loss.

The effect of the weighting factor $\alpha$ on the total amount of power and the total profit in case of price-II for the PBDEED problem is given in Table 5. It can be seen that, as the weighting factor $\alpha$ is increased (i.e., the importance of the emission is decreased), both the profit and the total power are increased.

Figures 11, 12, 13, and 14 show that the MPC closed-loop solutions asymptotically approach the optimal solutions of the PBDEED problem for price-II and for different values of $\alpha$. To show the IRP-MPC algorithm, we choose different

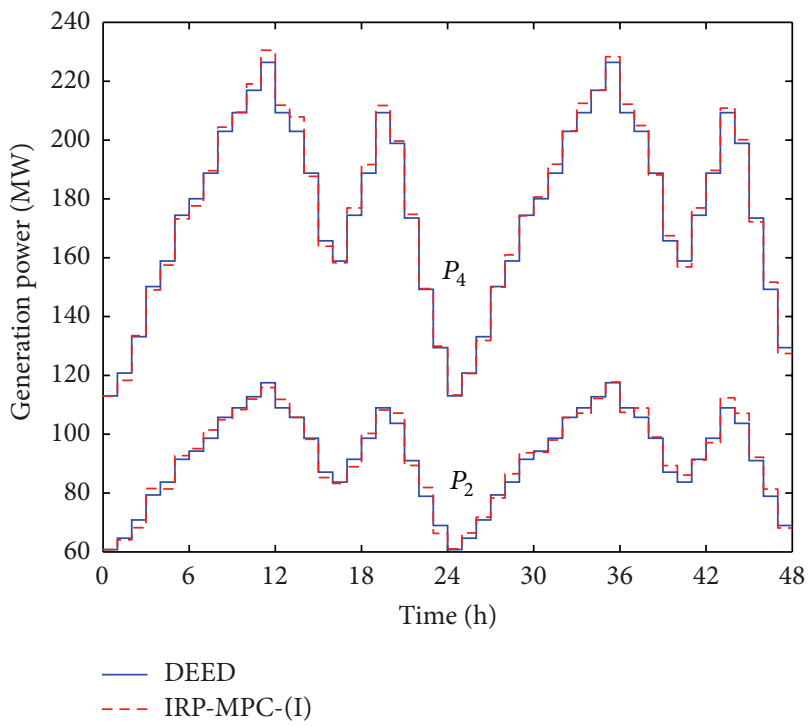

FIGURE 7: The generation output of the five-unit system under DEED $(\alpha=0.5)$ and IRP-MPC-(I).

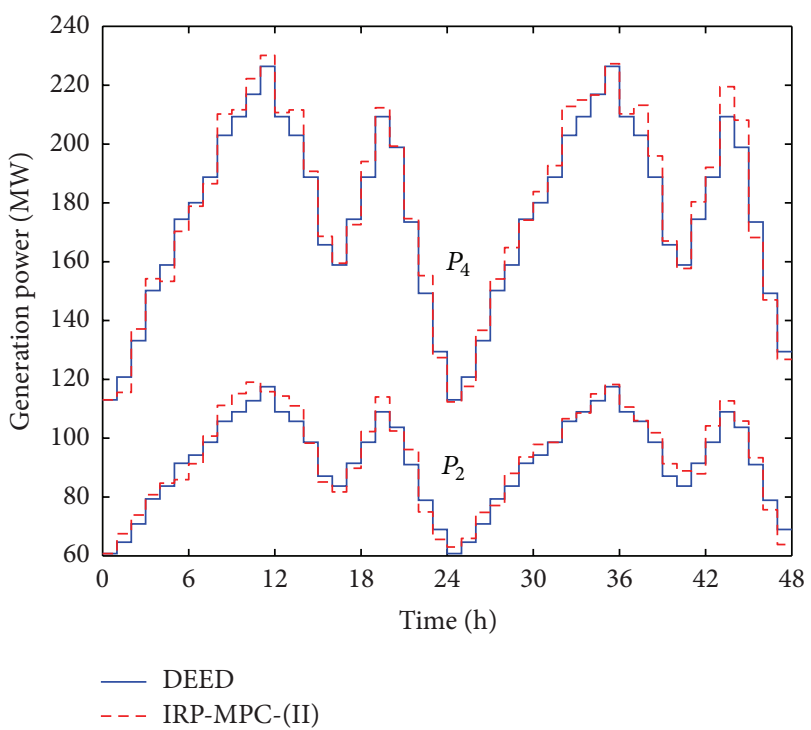

FIGURE 8: The generation output of the five-unit system under $\operatorname{DEED}(\alpha=0.5)$ and IRP-MPC-(II).

ranges of disturbances that arise from the execution of the optimal controller. We have two cases:

$$
\begin{aligned}
& \text { IRP-MPC-(I): } \varepsilon=(8,5,4,3,4,3), \\
& \text { IRP-MPC-(II): } \varepsilon=(32,20,16,12,16,12) .
\end{aligned}
$$

From Figures 15 and 16 we can see that, although the disturbance increased, the IRP-MPC-(II) still keep the disturbed system in the neighborhood of the optimal solution of the PBDEED problem. From these cases we observe that the size of this neighborhood depends on the bound of the disturbances. 


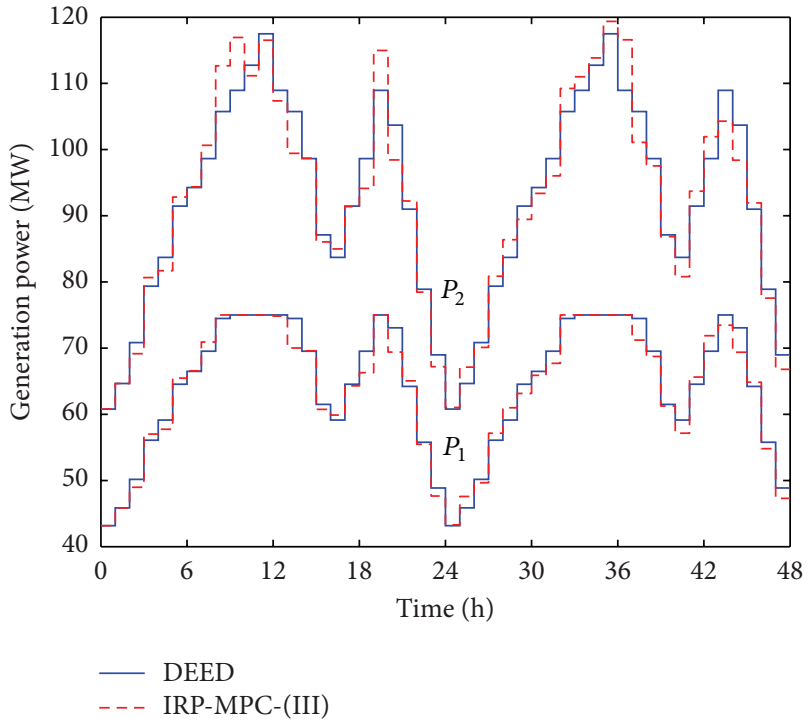

FIGURE 9: The generation output of the five-unit system under $\operatorname{DEED}(\alpha=0.5)$ and IRP-MPC-(III).

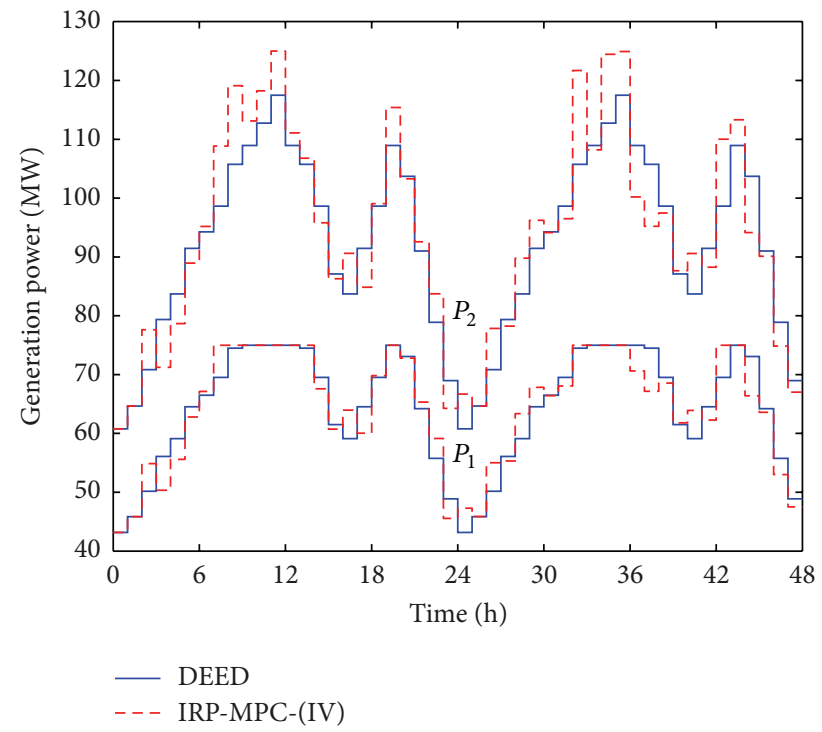

FIGURE 10: The generation output of the five-unit system under $\operatorname{DEED}(\alpha=0.5)$ and IRP-MPC-(IV).

Beside the convergence and robustness of the MPC, it has the following advantages [20].

(1) Reduced Dimensions. For the five-unit system, the DEED problem must solve an optimization problem with $5 \times 24=$ 120 variables. However, in each iteration step of the MPC algorithm, the algorithm solves an optimization problem with $5 \times 23=115$ number of variables which reduces 5 ( 6 for the six-unit system) dimensions in the optimization problem and makes the computation easier.

(2) Easy Implementation. Because of the MPC convergence, restarting the MPC algorithm from any time will give rise to

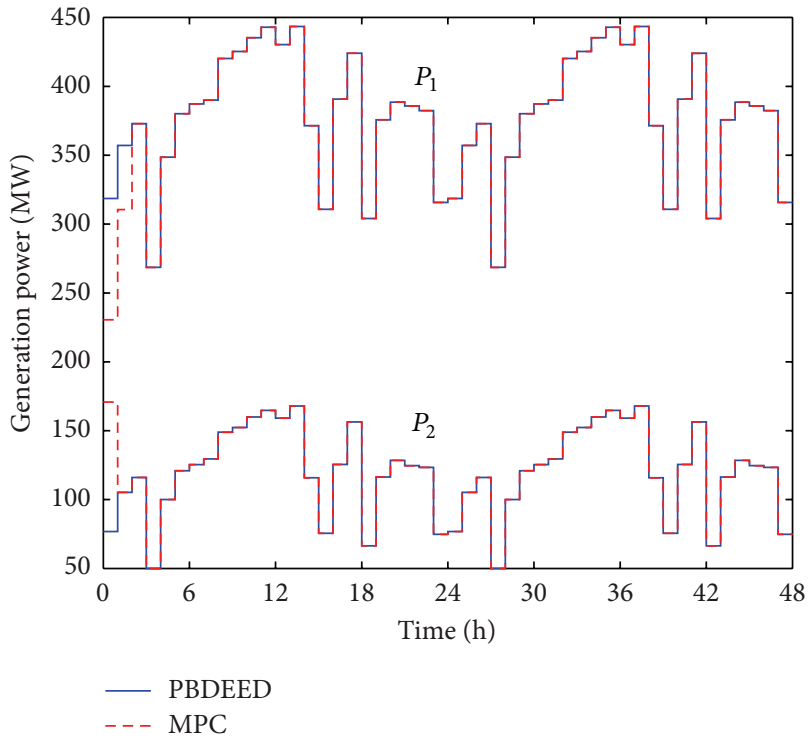

FIGURE 11: Convergence of the MPC solution of the six-unit system to those of PBDEED $(\alpha=1)$ and price-II.

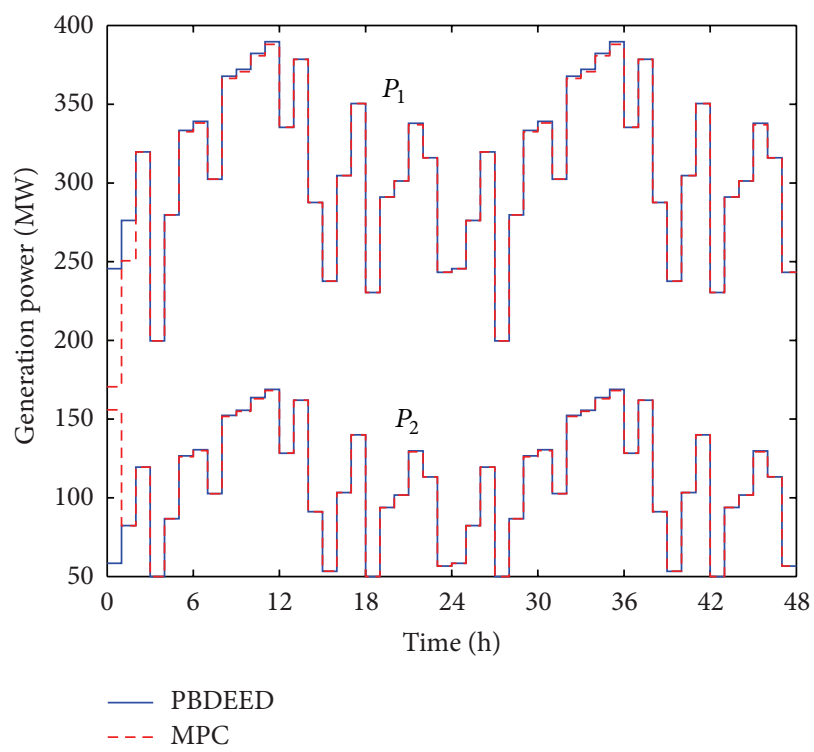

FIGURE 12: Convergence of the MPC solution of the six-unit system to those of PBDEED $(\alpha=0.7)$ and price-II.

the same convergence, which further implies that the MPC algorithm can be executed at any sampling time point. Thus the MPC algorithm is more favorable for practical applications than other open-loop algorithms [2].

\section{Conclusions}

In this paper, we have proposed a model predictive control approach for the periodic implementation of the optimal solutions of two optimal dynamic dispatch problems with emission and transmission losses. The first problem is the dynamic economic emission dispatch (DEED), and 


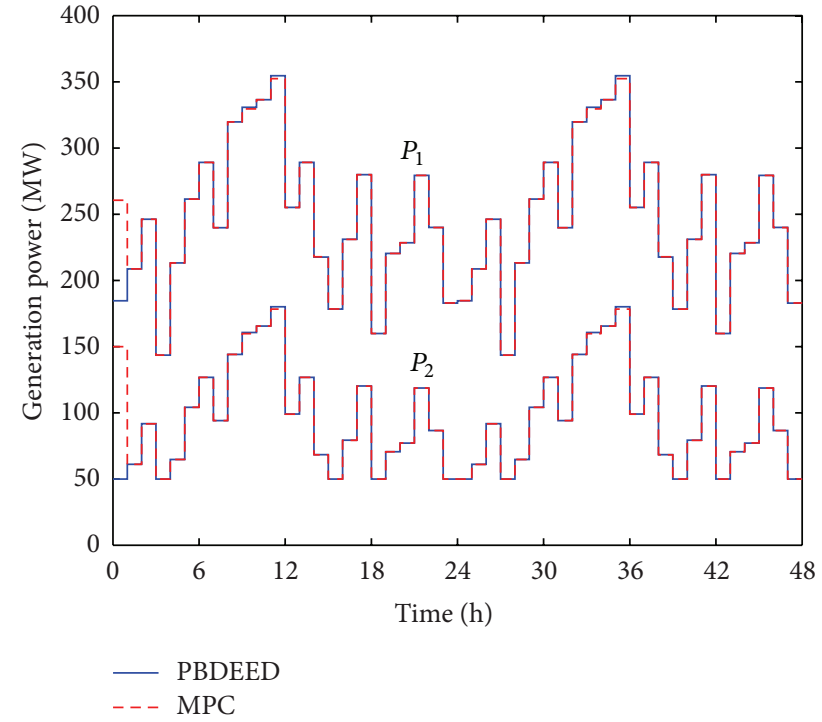

FIGURE 13: Convergence of the MPC solution of the six-unit system to those of PBDEED $(\alpha=0.5)$ and price-II.

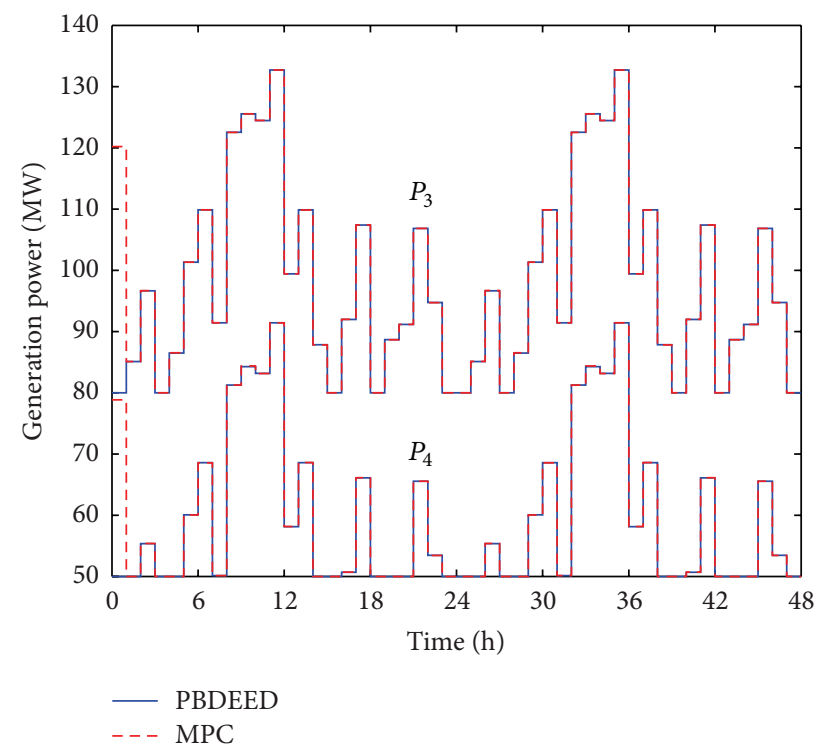

FIGURE 14: Convergence of the MPC solution of the six-unit system to those of PBDEED $(\alpha=0.2)$ and price-II.

the second is the profit-based dynamic economic emission dispatch (PBDEED). Both the demand and energy price are assumed to be periodic. We have assumed that there are certain disturbances or uncertainties in the execution of the optimal controller and in the forecasted demand. The convergence and robustness of the MPC algorithm are demonstrated through the application of MPC to the DEED problem with a five-unit system and to the PBDEED problem with a six-unit system.

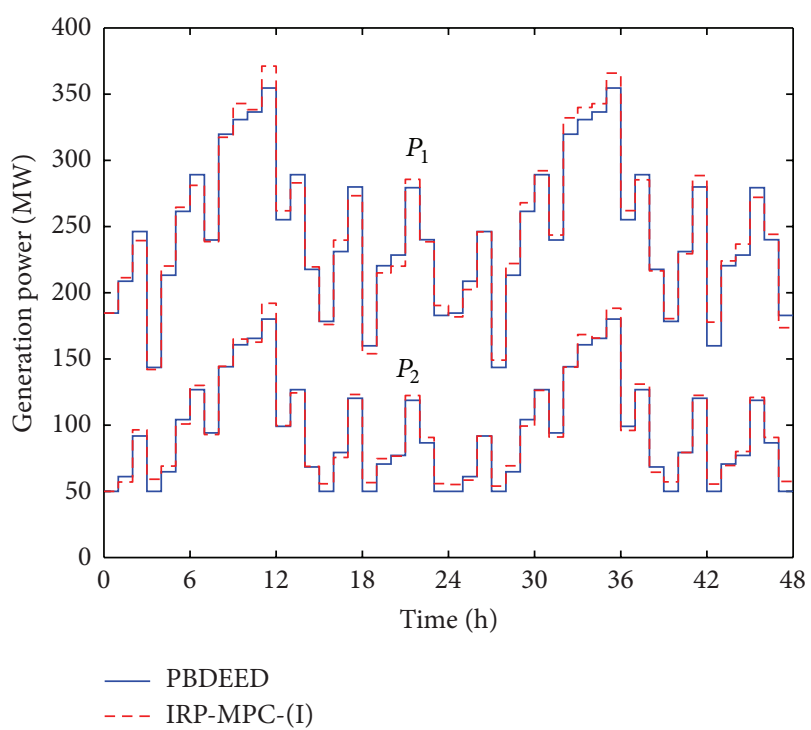

FIGURE 15: The generation output of the six-unit system under PBDEED $(\alpha=0.5)$, price-II, and IRP-MPC-(I).

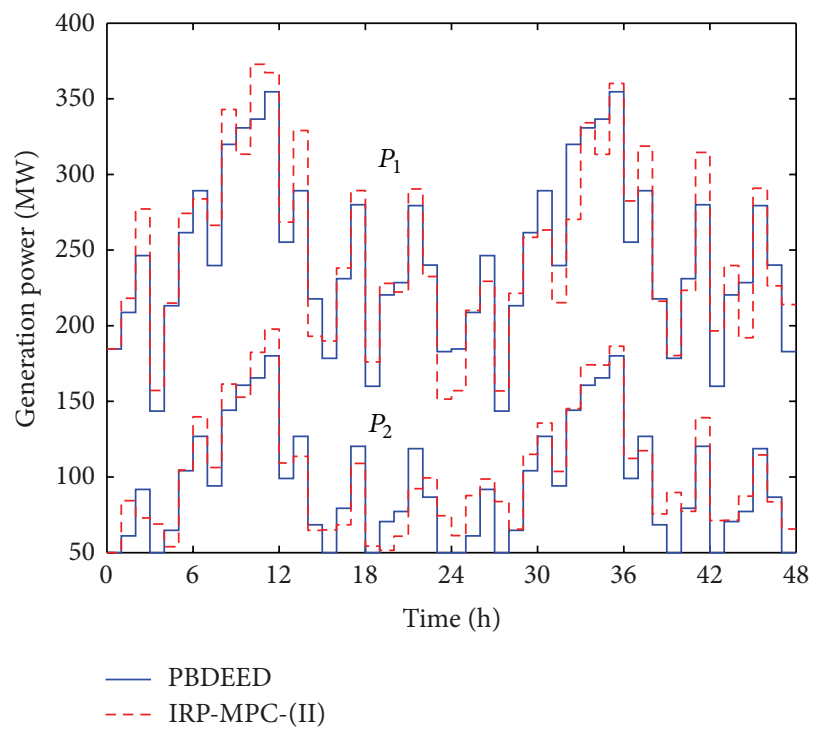

FIgURE 16: The generation output of the six-unit system under PBDEED $(\alpha=0.5)$, price-II, and IRP-MPC-(II).

\section{Appendix}

The transmission loss formula coefficients of the six-unit system are

$$
\begin{aligned}
B=10^{-4} & \left(\begin{array}{llllll}
0.420 & 0.051 & 0.045 & 0.057 & 0.078 & 0.066 \\
0.051 & 0.180 & 0.039 & 0.048 & 0.045 & 0.060 \\
0.045 & 0.039 & 0.195 & 0.051 & 0.072 & 0.057 \\
0.057 & 0.048 & 0.051 & 0.213 & 0.090 & 0.075 \\
0.078 & 0.045 & 0.072 & 0.090 & 0.207 & 0.096 \\
0.066 & 0.060 & 0.057 & 0.075 & 0.096 & 0.255
\end{array}\right) \text { per MW. }
\end{aligned}
$$




\section{Nomenclature}

$P_{i}^{t}$ : $\quad$ The power output of $i$ th unit at time $t$

$P_{i}^{\min }, P_{i}^{\max }$ : Lower and upper generation limits of the $i$ th unit

$\mathrm{UR}_{i}, \mathrm{DR}_{i}$ : Maximum ramp up/down rates for unit $i$

$C_{i}\left(P_{i}^{t}\right): \quad$ Generation cost of unit $i$ to produce $P_{i}^{t}$

$E_{i}\left(P_{i}^{t}\right): \quad$ Amount of emission for unit $i$ to produce $P_{i}^{t}$

$a_{i}, b_{i}, c_{i}: \quad$ Cost coefficients of unit $i$

$\alpha_{i}, \beta_{i}, \gamma_{i}$ : Emission coefficients of unit $i$

$u_{i}^{t}: \quad \quad$ Ramp rate of unit $i$ at time $t$

$N$ : $\quad$ Number of the intervals in the dispatch period

$n$ : $\quad$ Number of generating units

$D^{t}: \quad$ Load demand at time $t$

$\mathrm{SP}^{t}$ : $\quad$ Energy price at time $t$

$\alpha$ : Weighting factor

$B_{i j}$ : $\quad$ Loss formula coefficients.

\section{Acknowledgments}

This paper was funded by the Deanship of Scientific Research (DSR), King Abdulaziz University, Jeddah, under Grant no. 115/130/D1432. The authors, therefore, acknowledge with thanks DSR technical and financial support. The authors are grateful to the anonymous reviewers for constructive suggestions and valuable comments, which improved the quality of the paper.

\section{References}

[1] X. Xia and A. M. Elaiw, "Optimal dynamic economic dispatch: a review," Electric Power Systems Research, vol. 80, pp. 975-986, 2010.

[2] X. Xia, J. Zhang, and A. Elaiw, "An application of model predictive control to the dynamic economic dispatch of power generation," Control Engineering Practice, vol. 19, no. 6, pp. 638648, 2011.

[3] A. M. Elaiw, X. Xia, and A. M. Shehata, "Dynamic economic dispatch using hybrid DE-SQP for generating units with valvepoint effects," Mathematical Problems in Engineering, vol. 2012, Article ID 184986, 10 pages, 2012.

[4] P. Attaviriyanupap, H. Kita, E. Tanaka, and J. Hasegawa, "A hybrid EP and SQP for dynamic economic dispatch with nonsmooth fuel cost function," IEEE Transactions on Power Systems, vol. 17, no. 2, pp. 411-416, 2002.

[5] X. S. Han and H. B. Gooi, "Effective economic dispatch model and algorithm," International Journal of Electrical Power and Energy Systems, vol. 29, no. 2, pp. 113-120, 2007.

[6] T. A. A. Victoire and A. E. Jeyakumar, "A modified hybrid EPSQP approach for dynamic dispatch with valve-point effect," International Journal of Electrical Power and Energy Systems, vol. 27, no. 8, pp. 594-601, 2005.

[7] G. Irisarri, L. M. Kimball, K. A. Clements, A. Bagchi, and P. W. Davis, "Economic dispatch with network and ramping constraints via interior point methods," IEEE Transactions on Power Systems, vol. 13, no. 1, pp. 236-242, 1998.
[8] D. W. Ross and S. Kim, "Dynamic economic dispatch of generation," IEEE Transactions on Power Apparatus and Systems, vol. 99, no. 6, pp. 2060-2068, 1980.

[9] D. L. Travers and R. J. Kaye, "Dynamic dispatch by constructive dynamic programming," IEEE Transactions on Power Systems, vol. 13, no. 1, pp. 72-78, 1998.

[10] F. Li and R. K. Aggarwal, "Fast and accurate power dispatch using a relaxed genetic algorithm and a local gradient technique," Expert Systems with Applications, vol. 19, no. 3, pp. 159$165,2000$.

[11] P. Attaviriyanupap, H. Kita, E. Tanaka, and J. Hasegawa, "A fuzzy-optimization approach to dynamic economic dispatch considering uncertainties," IEEE Transactions on Power Systems, vol. 19, no. 3, pp. 1299-1307, 2004.

[12] J. H. Talaq, F. El-Hawary, and M. E. El-Hawary, "A summary of environmental/economic dispatch algorithms," IEEE Transactions on Power Systems, vol. 9, no. 3, pp. 1508-1516, 1994.

[13] M. Basu, "Dynamic economic emission dispatch using nondominated sorting genetic algorithm-II," International Journal of Electrical Power and Energy Systems, vol. 30, no. 2, pp. 140149, 2008.

[14] N. Pandit, A. Tripathi, S. Tapawsi, and M. Pandit, "An improved bacterial foraging algorithm for combined static/dynamic environmental economic dispatch," Applied Soft Computing, vol. 12, pp. 3500-3513, 2012.

[15] C. X. Guo, J. P. Zhan, and Q. H. Wu, "Dynamic economic emission dispatch based on group search optimizer with multiple producers," Electric Power Systems Research, vol. 86, pp. 8-16, 2012.

[16] M. Basu, "Particle swarm optimization based goal-attainment method for dynamic economic emission dispatch," Electric Power Components and Systems, vol. 34, no. 9, pp. 1015-1025, 2006.

[17] M. Basu, "Dynamic economic emission dispatch using evolutionary programming and fuzzy satisfying method," International Journal of Emerging Electric Power Systems, vol. 8, no. 4, article 1, 2007.

[18] J. S. Alsumait, M. Qasem, J. K. Sykulski, and A. K. Al-Othman, "An improved pattern search based algorithm to solve the dynamic economic dispatch problem with valve-point effect," Energy Conversion and Management, vol. 51, no. 10, pp. 20622067, 2010.

[19] A. Hooshmand, H. A. Malki, and J. Mohammadpour, "Power flow management of microgrid networks using model predictive control," Computers and Mathematics with Applications, vol. 64, no. 5, pp. 869-876, 2012.

[20] A. M. Elaiw, X. Xia, and A. M. Shehata, "Application of model predictive control to optimal dynamic dispatch of generation with emission limitations," Electric Power Systems Research, vol. 84, pp. 31-44, 2012.

[21] J. Zhang and X. Xia, "A model predictive control approach to the periodic implementation of the solutions of the optimal dynamic resource allocation problem," Automatica, vol. 47, no. 2, pp. 358-362, 2011.

[22] B. Otomega, A. Marinakis, M. Glavic, and T. Van Cutsem, "Model predictive control to alleviate thermal overloads," IEEE Transactions on Power Systems, vol. 22, no. 3, pp. 1384-1385, 2007.

[23] Y. H. Song and I. K. Yu, "Dynamic load dispatch with voltage security and environmental constraints," Electric Power Systems Research, vol. 43, no. 1, pp. 53-60, 1997. 
[24] J. P. S. Catalão, S. J. P. S. Mariano, V. M. F. Mendes, and L. A. F. M. Ferreira, "A practical approach for profitbased unit commitment with emission limitaions," International Journal of Electrical Power and Energy Systems, vol. 32, pp. 218-224, 2010.

[25] D. Q. Mayne, J. B. Rawlings, C. V. Rao, and P. O. M. Scokaert, "Constrained model predictive control: stability and optimality," Automatica, vol. 36, no. 6, pp. 789-814, 2000. 


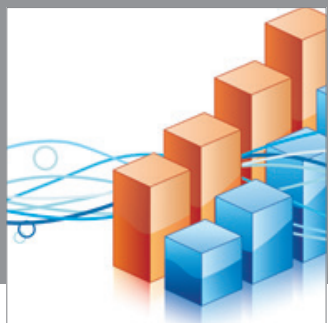

Advances in

Operations Research

mansans

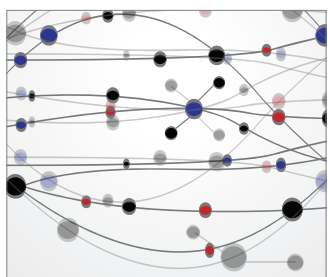

The Scientific World Journal
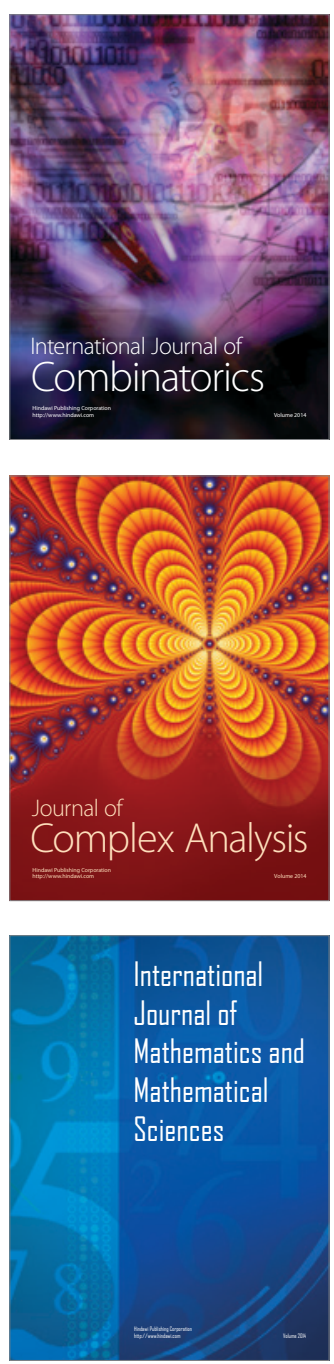
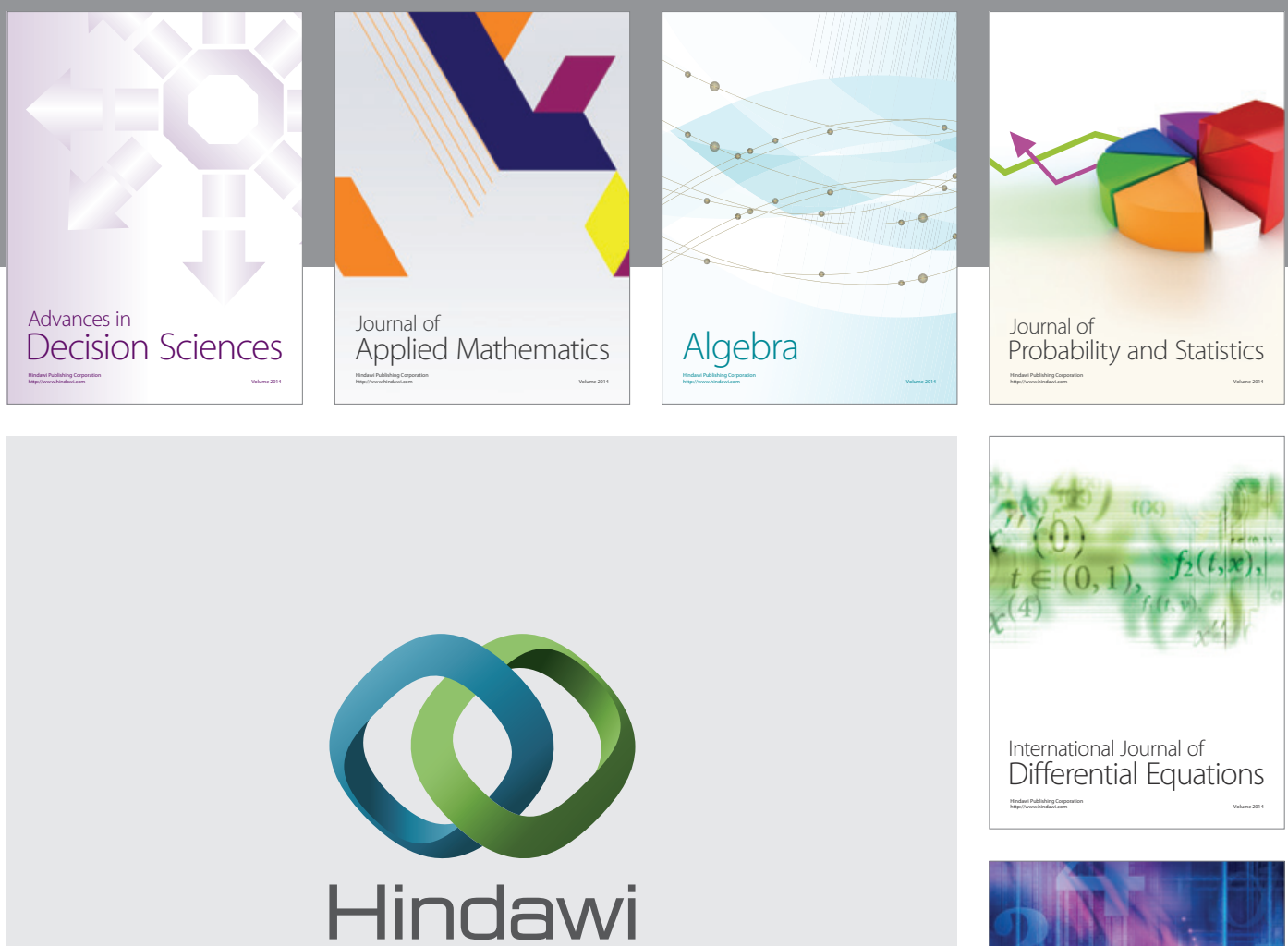

Submit your manuscripts at http://www.hindawi.com
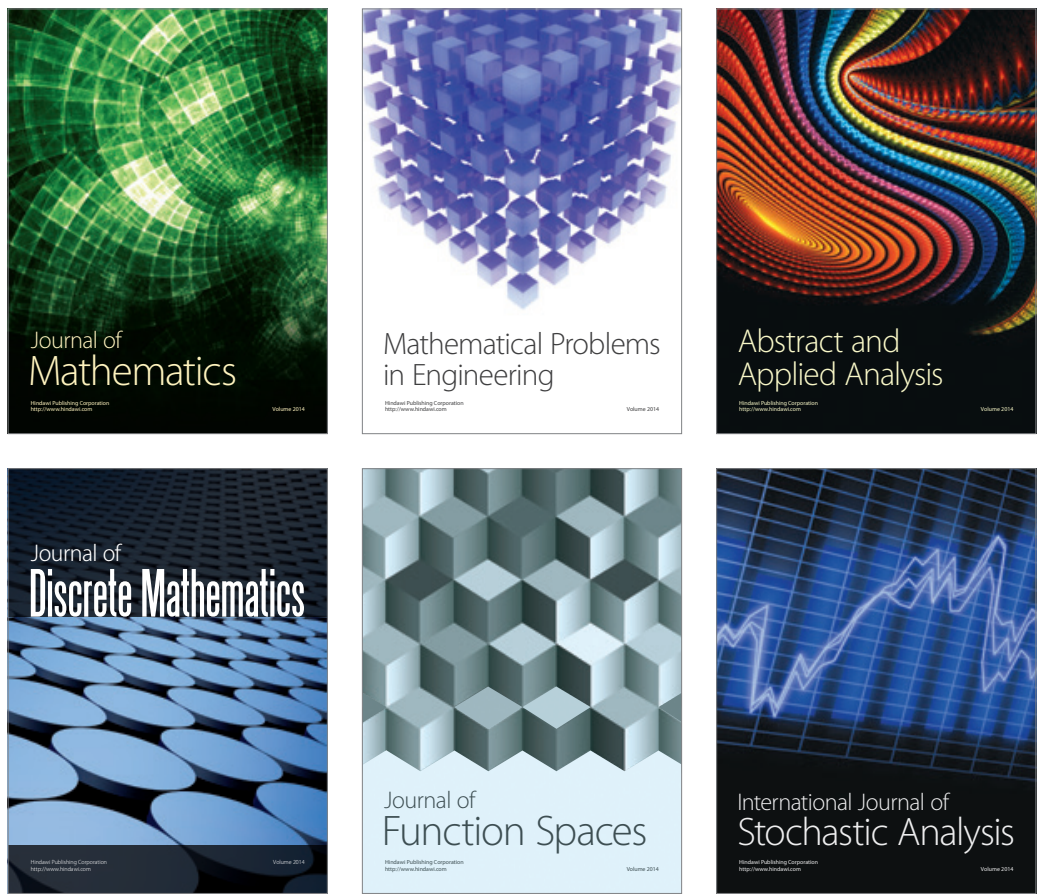

Journal of

Function Spaces

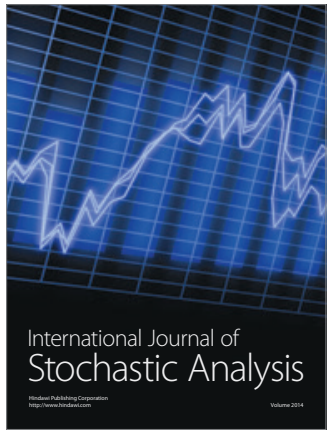

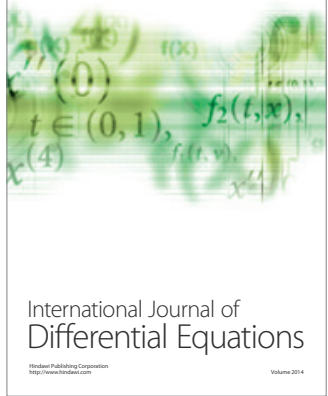
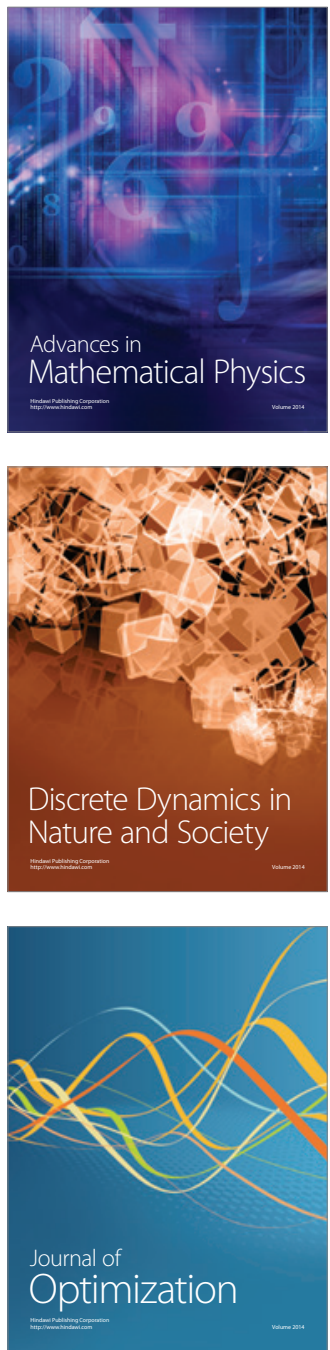